\title{
Diversidad florística en el complejo arqueológico La Bóveda, en el sur del departamento Amazonas, Perú
}

\author{
[Floristic diversity in the archaeological complex of La Boveda, in the south of \\ the Amazonas Departament, Peru]
}

\author{
Daniel Bernardo Montesinos-Tubée *1
}

\begin{abstract}
1. Universidad Nacional Autónoma de Chota (UNACH). Departamento Académico de Ciencias Forestales. Jr. José Osores No 418, 06120, Chota, Cajamarca, Perú. Correo electrónico: dbmtperu@gmail.com

(D. B. Montesinos-Tubée * Autor para correspondencia).
\end{abstract}

\begin{abstract}
Resumen
La vegetación del departamento de Amazonas (Perú) ha destacado por presentar una diversidad biológica bastante amplia y una alta importancia de los servicios ecosistémicos. Sin embargo, los ecosistemas son también amenazados por actividades forestales, agricultura o ganadería. Los objetivos del presente estudio fueron de evaluar la diversidad y composición de especies vasculares y helechos; comparar la composición de la diversidad según el hábito o tipo de planta y su distribución en pajonales y zonas boscosas; finalmente evaluar las tasas de endemismo según las zonas evaluadas en el ecosistema de Jalca. Un total de 294 especies, divididas en 190 géneros y 65 familias fueron registradas de diez diferentes estratos de vegetación en un área aproximada de $180000 \mathrm{~m}^{2}$ y a una elevación de 3400-3710 msnm. Se seleccionaron un total de 61 unidades de muestreo con dimensiones de $1-1000 \mathrm{~m}^{2}$ que variaron en tamaño según la cobertura vegetal y acceso topográfico. Los resultados demuestran que los pajonales poseen menor diversidad que las zonas boscosas. Mientras que a nivel de endemismo, las zonas boscosas registraron un leve aumento a comparación de los pajonales. Los resultados pueden relacionarse a la mayor humedad en las depresiones de origen kársticos y los parches boscosos no intervenidos, evidenciando la particularidad de la vegetación de las zonas boscosas en composición, diversidad y cobertura.
\end{abstract}

Palabras clave: Jalca, Kárstico, Pajonales, Parche boscoso, Vegetación.

\begin{abstract}
The vegetation of the department of Amazonas (Peru) has stood out for presenting a fairly wide biological diversity and a high importance of ecosystem services. However, ecosystems are also threatened by forestry, agriculture or livestock activities. The objectives of the present study were to evaluate the diversity and composition of vascular species and ferns; compare the composition of diversity according to habit or type of plant and its distribution in grasslands and wooded areas; and finally, evaluate the endemism rates according to the areas evaluated in the Jalca ecosystem. A total of 294 species, divided into 190 genera and 65 families were recorded from ten different vegetation strata in an approximate area of $180,000 \mathrm{~m}^{2}$ and at an elevation of 3400-3710 m. A total of 61 sampling units with dimensions of $1-1000 \mathrm{~m}^{2}$ were selected, which varied in size according to the vegetation cover and topographic access. The results show that the grasslands have less diversity than the wooded areas. While at the endemism level, the forested areas registered a slight increase compared to the grasslands. The results can be related to the higher humidity in the depressions of karst origin and the untouched forest patches, showing the particularity of the vegetation of the forested areas in composition, diversity and coverage.
\end{abstract}

Keywords: Grasslands, Jalca, Karst, Vegetation, Wooden patch. 


\section{INTRODUCCIÓN}

En referencia a los ecosistemas del sur del departamento de Amazonas del Perú, se encuentra poca información disponible tanto en literatura como en herbarios. Los esfuerzos realizados por Bussmann et al. (Expedición 2009-2013) en colectar material botánico ha sido vital para comprender mejor la estructura de la diversidad en el sur del departamento de Amazonas. En Perú, los conceptos de páramos del Norte o Jalca ha sido aplicado por Weberbauer (1945) y confirmado por Marcelo-Peña et al. (2006) los cuales se utilizan para describir la vegetación de Pajonal que tiene ausencia de árboles. Resulta complejo seleccionar si el ecosistema evaluado corresponde a la Jalca, confirmándose que parcialmente corresponde a este ecosistema siendo confirmado por sus características de ecosistema con abundancia de gramíneas pero con parches aislados densamente arbóreos. De acuerdo al mapa de zonificación del Perú (MINAM, 2009), el ecosistema evaluado, corresponde a las zonas de vida tipo bosque pluvial Montano Tropical (bp-MT) y páramo pluvial Subalpino Tropical (pp-SaT), extendiéndose entre los 3400 y 3710 msnm.

Diversos estudios han tratado la flora y vegetación asociada a restos arqueológicos, por ejemplo, Beresford-Jones et al. (2009) evaluaron los cambios ecológicos de largo plazo en las cuencas de los ríos Samaca y Ullujaya, valle bajo de Ica (costa sur del Perú). Otros estudios importantes incluyen Cárdenas-Martin y Milla-ViIlena (1980) para el valle de Ica, Rostworowski (1981) y Wernke (2003). Montesinos-Tubée (2016, 2017b) describe la vegetación en complejos arqueológicos de la cultura Yarowilca en el centro de Perú. Galán de Mera et al. (2020) describen como la distribución de sitios arqueológicos en Perú está relacionada con el clima y la vegetación natural. Schjellerup (2017) identifica algunas especies vegetales que fueron domesticadas en la localidad de Inka Llacta (en el contexto de la Provincia Inka de Chachapoyas) y que incluye el tomate de árbol (Solanum betaceum), la papaya (Carica sp.), el pajuro
(Fabaceae) y la ciruela de monte (Bunchosa armeniaca). Kauffmann (1980) describe brevemente algunas plantas halladas en exploración de ruinas en la selva del departamento de Amazonas. Rodríguez et al. (2015) describen las comunidades vegetales halladas en el Complejo Arqueológico Chan Chan, departamento de La Libertad. A la actualidad, no se conocen estudios realizados en amplitud que trate la vegetación en zonas arqueológicas de los Chachapoya a excepción del reciente estudio por Leiva et al. (2019) que destaca la diversidad florística del Complejo Arqueológico de Kuélap donde encontraron 147 especies florísticas. Schjellerup et al. (2003) hace referencia a la utilización de recursos, parte en la ceja de selva en el departamento de Amazonas. Torres et al. (2018) hace referencia a la etnobotánica de plantas medicinales utilizadas en comunidades adyacentes al Área de Conservación Privada San Antonio (Chachapoyas). La descripción de nuevas especies para el departamento de Amazonas es constante, teniendo como ejemplo algunas recientemente descritas por Damián (2019), Fernández-Hilario et al. (2020), Montesinos-Tubée (2017a), Montesinos-Tubée et al. (2019), Moonlight et al. (2020) y Romero-Hernández et al. (2019).

En el nororiente de Perú, se desarrolló la Cultura Chachapoyas (1000-1438 DC) (Kauffmann, 2017). Estudios paleoambientales hechos en la región de Chachapoyas están ayudando a esclarecer que la actividad humana en la zona inició durante el Holoceno (Church y Guengerich 2017). Los Chachapoyas ocuparon un territorio andino-amazónico con gran diversidad de flora y fauna, esto se relaciona con la congruencia de dos grandes ecosistemas: la selva alta (Yunga) y el bosque seco tropical, mientras que un tercero está relacionado con el antropismo y se le denomina "Jalca", o pastizales andinos (Kauffmann, 2017). Los asentamientos Chachapoyas están constituidos por casas de piedra en forma circular (Guengerich, 2014). Von Hagen (2016) confirma que dichos asentamientos formaban pequeñas aldeas que fluctuaban desde unas docenas de casas hasta pueblos con centenares de estructuras. Los Andes Amazónicos corres- 
ponden al flanco oriental de la cordillera de los Andes, el cual estuvo originalmente cubierto por densa vegetación tropical (Kauffmann, 2009). Guengerich (2014) determina que numerosas edificaciones han sido protegidas por la densa cobertura vegetal propia de la Selva Alta; los muros, aún preservados, permiten un examinar en detalle del espacio y materiales empleados en las edificaciones, esto a su vez, permite un análisis florístico a grandes rasgos de los sitios arqueológicos. A lo largo del proceso de deforestación al que fueron sometidos dichos ecosistemas, hoy en día la diversidad de flora y fauna ha variado considerablemente, la zona de estudio está representada por asentamientos con numerosas edificaciones rodeados por terrazas circulares que alguna vez fueron dedicadas al cultivo. Church y Guengerich (2017) indican que las contribuciones de estudios paleoambientales están proporcionando novedosa información sobre la cronología de ocupación y ecología humana en Chachapoyas prehispánica y que incluye el conocimiento de plantas cultivadas y la historia de prácticas de cultivo. Toyne y Anzellini (2017) identifican las características de los mauseleos de La Petaca, lugar cercano a la zona de estudio y que también se caracteriza por poseer mausoleos y construcciones de este tipo en murallas rocosas en la zona denominada Diablo Wasi.

En tanto, la zona estudiada comprende elementos de origen kárstico, que también son encontrados en el sureste de Cajamarca (Martorell, 1975) constan de cuarcitas y areniscas como ampliamente distribuidas en zonas tropicales de Sudamérica y las cuales están sujetas a procesos de disolución, generando cuevas significativas y kársticos (Auler, 2017). Arfé et al. (2017) estudió las cavidades kársticas halladas en Mina Grande (Amazonas, Perú) que constan de varias acumulaciones de minerales de óxido de Zinc, derivadas de la meteorización de un depósito de tipo "Mississippi Valley" ocurrido durante el Mesozoico de rocas carbonatadas en referencia a la Formación Condorsinga (Grupo Pucará). Las cavidades kársticas que albergan los minerales sin sulfuro se desarrollaron en el Mioceno a lo largo de fallas noroeste-sureste asociadas con estructuras regionales y localmente a lo largo de juntas de estratificación (Arfé et al., 2017).

Marcelo-Peña et al. (2006) afirma lo propuesto por Rodríguez (1996) indicando que la desaparición de los páramos está asociada a la explotación de recursos naturales, la deforestación de los bosques naturales, la quema y otras prácticas inapropiadas que provocan su destrucción y en consecuencia la erosión de suelos. En el presente estudio se aplica este concepto debido a que se comprueba la intensa quema de montes que se produce cada año en la zona evaluada que está correlacionada con los ecosistemas de Páramo. Los ecosistemas de Jalca son hábitats aislados y distintos en su riqueza de especies. El presente estudio se considera como el segundo trabajo que analiza la vegetación y diversidad florística en las inmediaciones de sitios arqueológicos en el departamento de Amazonas luego de la investigación presentada por Leiva et al. (2019). Se considera que las zonas altas del sur del departamento de Amazonas no han sido estudiadas a detalle y esto aún representa un vacío de información.

En este contexto, los objetivos del presente estudio son: (1) Identificar la diversidad y composición de especies vasculares y helechos en los restos arqueológicos de La Bóveda, Distrito Leymebamba, Provincia Chachapoyas, Departamento Amazonas, Perú, (2) comparar la composición de la diversidad según el hábito o tipo de planta y su distribución en pajonales y zonas boscosas, y (3) identificar las especies endémicas halladas en el estudio.

\section{MATERIALES Y MÉTODO}

\section{Área de estudio}

La presente investigación se realizó en los restos arqueológicos de La Bóveda; ubicado en el Distrito Leymebamba, Provincia Chachapoyas, Departamento Amazonas de la República del Perú; desde junio del 2015 a junio del 2018. Se encuentra localizado en Latitud Sur 649'53" - 650'26" y Longitud Oeste 77048'49" - 77049'24" (centroide), y a 3400-3710 msnm 


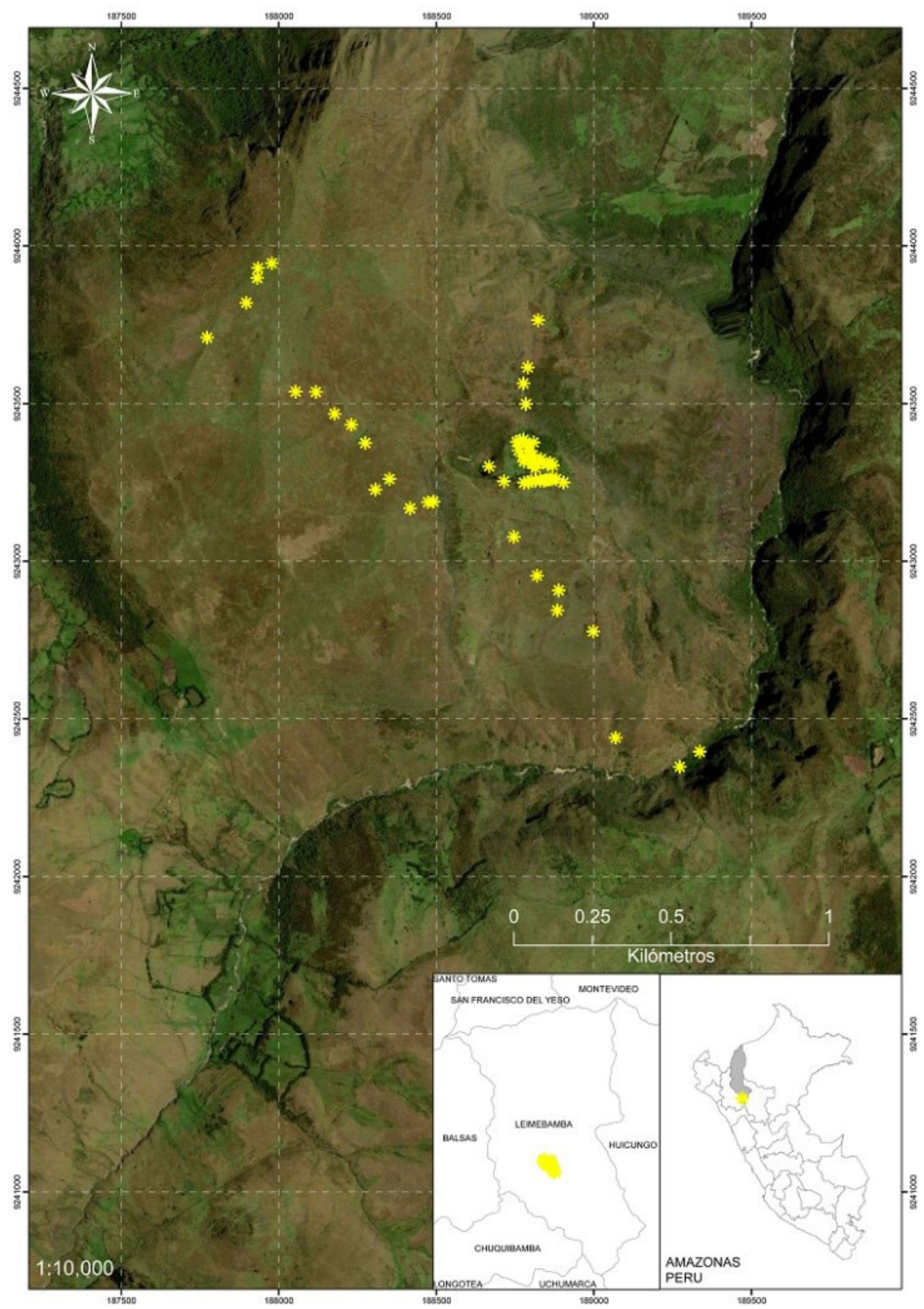

Figura 1. Ubicación de las 61 unidades de muestreo en el complejo arqueológico de La Boveda y alrededores en el sur del departamento de Amazonas, Perú. 
aproximadamente (Figura 1). La zona evaluada comprende un área aproximada de $180000 \mathrm{~m}^{2}$. La topografía es variada, con colinas y montañas, vertientes del Río Utcubamba, formada por materiales sedimentarios e ígneos (Escobedo, 2010). La precipitación promedio en la ciudad de Chachapoyas (Departamento de Amazonas) es de 778 mm/año (SENAMHI, 2018). De acuerdo a lo establecido por MINAM (2009), el ecosistema evaluado se clasifica como zona de transición entre los Andes y Bosques tropicales y corresponde a las zonas de vida tipo bosque pluvial Montano Tropical (bp-MT) y páramo pluvial Subalpino Tropical (pp-SaT) y extendiéndose entre los 3400 y 3710 msnm.

\section{Muestreo de la vegetación}

La evaluación se realizó a través del establecimiento de 61 parcelas que variaron en dimensiones de $1,2,4,6,9,12,16,36$ y $100 \mathrm{~m}^{2}$ para terrenos con ruinas arqueológicas enterradas, sub-enterradas o expuestas, al igual que en terrazas enterradas con cobertura de gramíneas. El tamaño de la unidad de evaluación para estos casos dependió de la topografía del terreno y el perímetro de la construcción incluyendo partes verticales y horizontales. Los análisis se realizaron en forma aleatoria, tomando como punto central las ruinas o sitios arqueológicos, a partir de ahí se analizó el perímetro de algunos restos, los interiores, exteriores, murales, techos y áreas contiguas en forma independiente una de otra. Por otro lado, en depresiones kársticas se tomó en consideración las especies que habitan en los alrededores y partes degradadas o erosionadas de manera natural, en estos casos las dimensiones fueron de 16 y 64 $\mathrm{m}^{2}, 1000 \mathrm{~m}^{2}$ en el caso de la depresión kárstica amplia. En zonas de riscos, las dimensiones de las unidades para toma de datos fueron de 100 y $225 \mathrm{~m}^{2}$. Finalmente, en quebradas secas se tomaron dimensiones de $1000 \mathrm{~m}^{2}$. Como variables medioambientales, se tomaron datos de elevación (msnm), pendiente (grados) y orientación. Los inventarios incluyeron las especies de Gimnospermas, Angiospermas y Pteridofitas. La presencia de especie fue anotada y la cobertura fue estimada en valor porcentual (Knapp, 1984; Mueller-Dombois y Ellenberg, 1974). Se aplicó el programa Twinspan (Hill, 1979) para reunir las 61 unidades en grupos según las similitudes de ocurrencia y cobertura de especies, posteriormente se definió manualmente las unidades que pertenecen a pajonales y zonas boscosas (según condiciones edafoclimáticas de las áreas, zonas con alta presencia de humedad en los suelos versus zonas con pajonales donde la retención de humedad es menor), a partir de ahí, se dividió en 10 tipos de estratos siguiendo los siguientes reglamentos pre-establecidos para esta investigación: a) Pajonales en ruinas arqueológicas enterradas, b) Pajonales con influencia de fuego, c) Cima de montaña, d) Vegetación en ruinas arqueológicas expuestas, e) Parche boscoso en zona arqueológica con reciente abandono, f) Vegetación en hundimientos kársticos, g) Vegetación en hundimiento kárstico profundo, h) Vegetación de quebradas ocasionalmente secas, i) Vegetación en ruinas arqueológicas con densa cobertura arbórea y j) Vegetación arbustiva en zona de riscos. Finalmente, los valores fueron transformados un único valor de presencia y ausencia que son los que se presentan en este trabajo. Ver figuras 3 a 11 para una mejor interpretación de la zona evaluada.

\section{Inventario florístico}

La identificación de especies se realizó en campo y especímenes no identificados fueron colectados siguiendo los permisos del Servicio Nacional Forestal y de Fauna Silvestre (SERFOR, Ministerio de Agricultura y Riego) N056-2016SERFOR/DGGSPFFS, N045-2017-SERFOR/ DGGSPFFS y N¹33-2018-MINAGRI-SERFOR/ DGGSPFFS para luego ser evaluados y depositados en herbarios (CPUN, HCSM, HSP, HUSA, HUT, MOL, USM; MO, B, F, LP, acrónimos de acuerdo a Thiers, 2016). El uso de herbario digital (JSTOR, Neotropical Herbarium Specimens - The Field Museum y Tropicos - Missouri Botanical Garden) fue indispensable para la identificación de especímenes. La nomenclatura de las especies está de acuerdo con Brako y Zarucchi (1993), las versiones electrónicas de Tropicos (2020), IPNI (2014), JSTOR (2013) y The Plant List (2010). 
Tabla 1. Lista total de 56 endemismos (León et al., 2006; Montesinos-Tubée, 2018; Tropicos, 2020), hallados en el complejo arqueológico de La Boveda donde el valor 1 se refiere a la presencia de la especie en la zona evaluada.

\begin{tabular}{|c|c|c|c|c|c|c|c|c|c|c|c|}
\hline \multirow{4}{*}{ Familia } & \multirow{4}{*}{$\begin{array}{l}\text { Área } \\
\text { Número de orden } \\
\% \text { endemismo sobre el total de especies } \\
\text { Nombre científico / Número de especies }\end{array}$} & \multicolumn{4}{|c|}{ Pajonales } & \multicolumn{6}{|c|}{ Zonas boscosas } \\
\hline & & 1 & 2 & 3 & 4 & 5 & 6 & 7 & 8 & 9 & 10 \\
\hline & & 3,06 & 3,74 & 4,76 & 5,1 & 4,08 & 3,74 & 6,46 & 5,44 & 8,5 & 4,76 \\
\hline & & 9 & 11 & 14 & 15 & 12 & 11 & 19 & 16 & 25 & 14 \\
\hline Alstroemeriaceae & Bomarea goniocaulon Baker & & & & & & & $\mathrm{x}$ & $\mathrm{x}$ & & \\
\hline Asteraceae & $\begin{array}{l}\text { Aristeguietia pseudarborea (Hieron.) R.M. } \\
\text { King \& H. Rob. }\end{array}$ & & & & & & & & $\mathrm{x}$ & & \\
\hline Asteraceae & $\begin{array}{l}\text { Baccharis cf. brachylaenoides var. mathewsii } \\
\text { Cuatrec. }\end{array}$ & $\mathrm{x}$ & $x$ & & $\mathrm{x}$ & $\mathrm{x}$ & $x$ & & & $x$ & \\
\hline Asteraceae & Baccharis ledifolia Kunth & & $x$ & & & $x$ & & & & & \\
\hline Asteraceae & Baccharis woytkowskii Joch. Müll. & & & $x$ & $x$ & $x$ & & $x$ & & & $\mathrm{x}$ \\
\hline Asteraceae & Coreopsis lopez-mirandae Sagást. & & & $x$ & & & & & & & $x$ \\
\hline Asteraceae & Coreopsis sherffii S.F. Blake & & & & & & & & $\mathrm{x}$ & & \\
\hline Asteraceae & Cronquistianthus bishopii R.M. King \& H. Rob. & & & & & & & $x$ & & $\mathrm{x}$ & $\mathrm{x}$ \\
\hline Asteraceae & Dendrophorbium chopinii Montesinos & $\mathrm{x}$ & $\mathrm{x}$ & & $\mathrm{x}$ & $\mathrm{x}$ & & $x$ & $\mathrm{x}$ & $\mathrm{x}$ & $\mathrm{x}$ \\
\hline Asteraceae & $\begin{array}{l}\text { Dendrophorbium usgorense (Cuatrec.) C. Je- } \\
\text { ffrey }\end{array}$ & & & & $\mathrm{x}$ & & & $x$ & & $\mathrm{x}$ & \\
\hline Asteraceae & $\begin{array}{l}\text { Dorobaea callacallensis (Cuatrec.) B. Nord. \& } \\
\text { Pruski }\end{array}$ & & $\mathrm{x}$ & $\mathrm{x}$ & & & $\mathrm{x}$ & & & & \\
\hline Asteraceae & Gynoxys caracensis Muschl. & & & & & & & & & $\mathrm{x}$ & \\
\hline Asteraceae & Gynoxys cerrateana B. Herrera & & & & $\mathrm{x}$ & & $\mathrm{x}$ & $\mathrm{x}$ & $\mathrm{x}$ & $x$ & \\
\hline Asteraceae & Gynoxys soukupii Cuatrec. & & & & & & & $x$ & & $x$ & $x$ \\
\hline Asteraceae & Jungia schuerae Harling & & & & & $\mathrm{x}$ & & $x$ & $\mathrm{x}$ & $x$ & \\
\hline Asteraceae & Munnozia lyrata (A. Gray) H. Rob. \& Brettell & & & & & & $x$ & $x$ & & $x$ & \\
\hline Asteraceae & Oritrophium hirtopilosum (Hieron.) Cuatrec. & & & $x$ & $x$ & & & & $x$ & & $x$ \\
\hline Asteraceae & Pappobolus stuebelii (Hieron.) Panero & & & $x$ & & $x$ & & & & & \\
\hline Asteraceae & Senecio coymolachensis Cabrera & & & & & & & & $\mathrm{x}$ & & \\
\hline Asteraceae & Senecio kingbishopii Cuatrec. & $x$ & & & & $x$ & & & & $x$ & \\
\hline Asteraceae & Senecio minesinus Cuatrec. & & & & & & & & & $\mathrm{x}$ & \\
\hline Asteraceae & $\begin{array}{l}\text { Senecio repens Stokes var. macbridei (Cua- } \\
\text { trec.) Cabrera }\end{array}$ & & $x$ & & & & & & & & \\
\hline Asteraceae & Stevia puberula Hook. & & $x$ & $x$ & & $x$ & & & & & $x$ \\
\hline Asteraceae & Verbesina cf. ochroleucotricha Sagást. & & & & & & & $x$ & & $x$ & \\
\hline Bromeliaceae & Puya cf. fulgens L.B. Sm. & & & & & & & & & & $x$ \\
\hline Bromeliaceae & Tillandsia cf. ferreyrae L.B. Sm. & & & & & & & & $x$ & & \\
\hline Calceolariaceae & Calceolaria bicrenata Ruiz \& Pav. & & & & & & & & & $\mathrm{x}$ & \\
\hline Calceolariaceae & Calceolaria concava Molau & & & & & & & $x$ & & & \\
\hline Calceolariaceae & Calceolaria dentifolia Edwin & & & & & $x$ & & & & & \\
\hline Calceolariaceae & Calceolaria linearis Ruiz \& Pav. & & & & $x$ & & $x$ & & & & \\
\hline Calceolariaceae & Calceolaria rufescens Molau & & & $x$ & & $\mathrm{x}$ & & $x$ & & $\mathrm{x}$ & $x$ \\
\hline Campanulaceae & Siphocampylus matthiaei A. DC. & & & & & & & & & $\mathrm{x}$ & \\
\hline Caryophyllaceae & Drymaria glaberrima Bartl. & & & & & & & & $x$ & & \\
\hline Caryophyllaceae & Drymaria grandiflora Bartl. & & & & & & & $x$ & $x$ & & \\
\hline Caryophyllaceae & Paronychia macbridei Chaudhri & & & $\mathrm{x}$ & & & & $x$ & & & \\
\hline Crassulaceae & $\begin{array}{l}\text { Sedum reniforme (H. Jacobsen) Thiede \& 't } \\
\text { Hart }\end{array}$ & & & $x$ & $x$ & & & & & & \\
\hline Fabaceae & Lupinus peruvianus Ulbr. & $\mathrm{x}$ & $\mathrm{x}$ & & $\mathrm{x}$ & & $\mathrm{x}$ & & & $\mathrm{x}$ & \\
\hline Gentianaceae & Gentianella decemnectaria J.S. Pringle & $x$ & $x$ & $x$ & $x$ & & & & & $x$ & $x$ \\
\hline Geraniaceae & Geranium peruvianum Hieron. & $x$ & & $x$ & $x$ & $\mathrm{x}$ & $\mathrm{x}$ & $x$ & & $x$ & $x$ \\
\hline Lamiaceae & Clinopodium argenteum (Kunth) Govaerts & & & & & $x$ & & & & & $x$ \\
\hline Lamiaceae & Salvia camporum Epling & & & & & & & $x$ & & $x$ & \\
\hline Lamiaceae & Salvia striata Benth. & & & & & & & & $x$ & & \\
\hline Loasaceae & Nasa basilica T. Henning \& Weigend & & & & & & & $x$ & & $x$ & \\
\hline Melastomataceae & Brachyotum figueroae J.F. Macbr. & & & & & & & $x$ & & & \\
\hline Onagraceae & Fuchsia mathewsii J.F. Macbr. & & & & & & & & $x$ & $x$ & \\
\hline Orchidaceae & Stelis parviflora (Ruiz \& Pav.) Pers. & & & & & & & & $x$ & & \\
\hline
\end{tabular}




\begin{tabular}{|c|c|c|c|c|c|c|c|c|c|c|c|}
\hline \multirow{4}{*}{ Familia } & \multirow{4}{*}{$\begin{array}{l}\text { Área } \\
\text { Número de orden } \\
\% \text { endemismo sobre el total de especies } \\
\text { Nombre científico }\end{array}$} & \multicolumn{4}{|c|}{ Pajonales } & \multicolumn{6}{|c|}{ Zonas boscosas } \\
\hline & & 1 & 2 & 3 & 4 & 5 & 6 & 7 & 8 & 9 & 10 \\
\hline & & 3,06 & 3,74 & 4,76 & 5,1 & 4,08 & 3,74 & 6,46 & 5,44 & 8,5 & 4,76 \\
\hline & & 9 & 11 & 14 & 15 & 12 & 11 & 19 & 16 & 25 & 14 \\
\hline Orobanchaceae & Castilleja peruviana T.I. Chuang \& Heckard & & & & $\mathrm{x}$ & & & & & & \\
\hline Orobanchaceae & Castilleja vadosa T.I. Chuang \& Heckard & & $x$ & $x$ & $x$ & & & & & & \\
\hline Piperaceae & Peperomia andina Pino & & & & & & $x$ & & & & \\
\hline Piperaceae & Peperomia crystallina Ruiz \& Pav. & & & & & & & & & $x$ & \\
\hline Poaceae & Festuca glabrata Tovar & $\mathrm{x}$ & $\mathrm{x}$ & $x$ & $x$ & & $x$ & $\mathrm{x}$ & $x$ & $x$ & $x$ \\
\hline Pteridaceae & Cheilanthes cf. peruviana (Desv.) T. Moore & & & & & & & & $x$ & $x$ & \\
\hline Rosaceae & Polylepis multijuga Pilg. & & & & & & & & & $x$ & \\
\hline Scrophulariaceae & Alonsoa minor Edwin & & & & & & $x$ & & & & \\
\hline Urticaceae & Pilea dombeyana Wedd. & $x$ & & & & & & & & & \\
\hline Verbenaceae & Stachys peruviana Dombey ex Benth. & $x$ & $\mathrm{x}$ & $x$ & $x$ & & $x$ & & & & $x$ \\
\hline
\end{tabular}

Tabla 2. Especies de plantas registradas en las zonas de evaluación en el complejo arqueológico de La Bóveda y alrededores, en el departamento de Amazonas, norte de Perú según el hábito u tipo de planta. El estatus se refiere a N: Nativo, E: Endémico, I: Introducido, - sin datos debido a falta de identificación. Los valores referidos a Pajonales corresponde a: 1. Pajonales en ruinas arqueológicas enterradas, 2. Pajonales con influencia de fuego, 3. Cima de montaña y 4 . Vegetación en ruinas arqueológicas expuestas; en tanto que para parches boscosos se identifican los siguientes: 5. Parche boscoso en zona arqueológica con reciente abandono, 6. Vegetación en hundimientos kársticos, 7. Vegetación en hundimiento kárstico profundo, 8. Vegetación de quebradas ocasionalmente secas, 9. Vegetación en ruinas arqueológicas con densa cobertura arbórea y 10 . Vegetación arbustiva en zona de riscos. El valor numérico por especie corresponde a presencia.

\begin{tabular}{|c|c|c|c|c|c|c|c|c|c|c|c|c|}
\hline \multirow{2}{*}{ Familia } & \multirow{2}{*}{ Nombre Científico } & \multirow{2}{*}{ Estatus } & \multicolumn{4}{|c|}{ Pastizales } & \multicolumn{6}{|c|}{ Parches boscosos } \\
\hline & & & L1 & $\mathbf{L} 2$ & $\mathbf{L 3}$ & L4 & $\mathbf{L 5}$ & $\mathbf{L 6}$ & L7 & $\mathbf{L 8}$ & L9 & L10 \\
\hline \multicolumn{13}{|l|}{ Árboles } \\
\hline Adoxaceae & Sambucus peruviana Kunth & $\mathrm{N}$ & & & & & & & & & $x$ & \\
\hline Asteraceae & Gynoxys sp. & - & & & & & & & & & $x$ & \\
\hline Asteraceae & Gynoxys caracensis Muschl. & $\mathrm{E}$ & & & & $\mathrm{x}$ & & $x$ & $\mathrm{x}$ & $\mathrm{x}$ & $x$ & \\
\hline Asteraceae & Gynoxys cerrateana B. Herrera & $\mathrm{E}$ & & & & & & & $\mathrm{x}$ & & $x$ & $x$ \\
\hline Asteraceae & Gynoxys soukupii Cuatrec. & $\mathrm{E}$ & & & & & $x$ & & & & & \\
\hline Asteraceae & Jungia schuerae Harling & $\mathrm{E}$ & & & & & $x$ & & $\mathrm{x}$ & $\mathrm{x}$ & $\mathrm{x}$ & \\
\hline Asteraceae & Verbesina cf. ochroleucotricha Sagást. & $\mathrm{E}$ & & & & & & & $x$ & & $x$ & \\
\hline Asteraceae & Verbesina cumingii (Griseb.) S.F.Blake & $\mathrm{N}$ & & & & & & & & $x$ & & \\
\hline Asteraceae & Pentacalia peruviana (Pers.) Cuatrec. & $\mathrm{N}$ & & & & & & & & & $\mathrm{x}$ & \\
\hline Campanulaceae & Siphocampylus matthiaei A. DC. & $E$ & & & & & & & & $\mathrm{x}$ & & \\
\hline Elaeocarpaceae & Vallea stipularis L. f. & $\mathrm{N}$ & & & & $x$ & & & $\mathrm{x}$ & $x$ & & $x$ \\
\hline Escalloniaceae & Escallonia sp. & - & & & & & & & & $x$ & & $x$ \\
\hline Fabaceae & Otholobium mexicanum (L. f.) J.W. Grimes & $\mathrm{N}$ & & $x$ & & & & $x$ & $\mathrm{x}$ & & $x$ & \\
\hline Loganiaceae & Buddleja incana Ruiz \& Pav. & $\mathrm{N}$ & & & & & $x$ & & $x$ & & & \\
\hline Rosaceae & Polylepis multijuga Pilg. & $\mathrm{E}$ & & & & & & & & $\mathrm{x}$ & & \\
\hline Rosaceae & Hesperomeles cuneata Lindl. & $\mathrm{N}$ & & & & & & & & & $\mathrm{x}$ & \\
\hline Rosaceae & Hesperomeles ferruginea (Pers.) Benth. & $\mathrm{N}$ & & & & & & & & $\mathrm{x}$ & & \\
\hline Solanaceae & Solanum barbulatum Zahlbr. & $\mathrm{N}$ & & & & & & & $\mathrm{x}$ & $\mathrm{x}$ & & \\
\hline Solanaceae & Solanum sanchez-vegae S. Knapp & $\mathrm{N}$ & & & & & & & & & $x$ & \\
\hline \multicolumn{13}{|l|}{ Arbustos } \\
\hline Asteraceae & Aetheolaena sp. & - & & & & & $\mathrm{x}$ & & $\mathrm{x}$ & & & \\
\hline Asteraceae & $\begin{array}{l}\text { Ageratina articulata (Sch. Bip. ex Hieron.) R.M. King \& } \\
\text { H. Rob. }\end{array}$ & $\mathrm{N}$ & $x$ & & & & $\mathrm{x}$ & & & & & \\
\hline Asteraceae & Ageratina sp. & - & & & & $\mathrm{x}$ & & & $x$ & & & \\
\hline
\end{tabular}




\begin{tabular}{|c|c|c|c|c|c|c|c|c|c|c|c|c|}
\hline \multirow{2}{*}{ Familia } & \multirow{2}{*}{ Nombre Científico } & \multirow{2}{*}{ Estatus } & \multicolumn{4}{|c|}{ Pastizales } & \multicolumn{6}{|c|}{ Parches boscosos } \\
\hline & & & L1 & L2 & L3 & L4 & $\mathbf{L 5}$ & L6 & L7 & L8 & L9 & L10 \\
\hline Asteraceae & $\begin{array}{l}\text { Aristeguietia pseudarborea (Hieron.) R.M. King \& H. } \\
\text { Rob. }\end{array}$ & $\mathrm{E}$ & & & & $x$ & $x$ & & & $x$ & & \\
\hline Asteraceae & Baccharis cf. brachylaenoides var. mathewsii Cuatrec. & $\mathrm{E}$ & $x$ & $x$ & $x$ & & & $x$ & & & $x$ & $x$ \\
\hline Asteraceae & Baccharis emarginata (Ruiz \& Pav.) Pers. & $\mathrm{N}$ & & & & & & & & & $x$ & \\
\hline Asteraceae & Baccharis genistelloides (Lam.) Pers. & $\mathrm{N}$ & & & & & & $x$ & & & & \\
\hline Asteraceae & Baccharis latifolia (Ruiz \& Pav.) Pers. & $\mathrm{N}$ & & & & & & & $x$ & & & \\
\hline Asteraceae & Baccharis ledifolia Kunth & $\mathrm{E}$ & & & & & & & & $x$ & & \\
\hline Asteraceae & Baccharis peruviana Cuatrec. & $\mathrm{N}$ & $x$ & $x$ & & $x$ & $x$ & $x$ & & & $x$ & \\
\hline Asteraceae & Baccharis phylicoides Kunth & $\mathrm{N}$ & & $x$ & & & $x$ & & & & & \\
\hline Asteraceae & Baccharis sinuata Kunth & $\mathrm{N}$ & & & $x$ & $x$ & $x$ & & $x$ & & & $x$ \\
\hline Asteraceae & Baccharis woytkowskii Joch. Müll. & $\mathrm{E}$ & & & & & & & & & $x$ & \\
\hline Asteraceae & Barnadesia arborea Kunth & $\mathrm{N}$ & & & & & & & $x$ & & & \\
\hline Asteraceae & Chromolaena leptocephala (DC.) R.M. King \& H. Rob. & $\mathrm{N}$ & & & & & $x$ & & & & & \\
\hline Asteraceae & Coreopsis lopez-mirandae Sagást. & $\mathrm{E}$ & & & & $x$ & & $x$ & & & & \\
\hline Asteraceae & Coreopsis sherffii S.F. Blake & $E$ & & & $x$ & & $x$ & & $x$ & & $x$ & $x$ \\
\hline Asteraceae & Cronquistianthus bishopii R.M. King \& H. Rob. & $\mathrm{E}$ & & & & & $x$ & & & & & $x$ \\
\hline Asteraceae & Diplostephium callaense Cuatrec. & $\mathrm{N}$ & & & $x$ & & & & & & & $x$ \\
\hline Asteraceae & $\begin{array}{l}\text { Eupatorium exerto-venosum var. pseudofastigiatum } \\
\text { B.L. Rob. }\end{array}$ & $\mathrm{N}$ & & & & & & & & $x$ & & \\
\hline Asteraceae & Ferreyranthus sp. & $\mathrm{N}$ & & & & & & & $x$ & & $x$ & $x$ \\
\hline Asteraceae & Jungia rugosa Less. & $\mathrm{N}$ & & & & & & & & $x$ & $x$ & \\
\hline Asteraceae & Loricaria leptothamna (Mattf.) Cuatrec. & $\mathrm{N}$ & & & $x$ & & $x$ & & & & & \\
\hline Asteraceae & Pappobolus stuebelii (Hieron.) Panero & $\mathrm{E}$ & & & & & & & & & & $x$ \\
\hline Asteraceae & Pentacalia peruviana (Pers.) Cuatrec. & $\mathrm{N}$ & & & & & & & $x$ & & $x$ & \\
\hline Asteraceae & Senecio kingbishopii Cuatrec. & $\mathrm{E}$ & & & & & & & & $x$ & & \\
\hline Asteraceae & Senecio minesinus Cuatrec. & $E$ & $x$ & & & & $x$ & & & & $x$ & \\
\hline Asteraceae & Senecio sp. 1 & - & & & & & & & & & $x$ & \\
\hline Asteraceae & Senecio sp. 2 & - & & $x$ & $x$ & $x$ & & & $x$ & $x$ & $x$ & \\
\hline Asteraceae & Senecio sp. 3 & - & & & & & & & & & & $x$ \\
\hline Asteraceae & Senecio sp. 4 & - & & $x$ & & $x$ & & & & & & \\
\hline Bromeliaceae & Puya cf. fulgens L.B. Sm. & $E$ & $x$ & & $x$ & $x$ & & $x$ & $x$ & & $x$ & $x$ \\
\hline Calceolariaceae & Calceolaria atahualpae Kraenzl. & $\mathrm{N}$ & & & & & & & $x$ & & $x$ & \\
\hline Calceolariaceae & Calceolaria bicrenata Ruiz \& Pav. & $\mathrm{E}$ & & $x$ & & $x$ & & & $x$ & & $x$ & $x$ \\
\hline Calceolariaceae & Calceolaria concava Molau & $E$ & & & & & & & $x$ & $x$ & & \\
\hline Calceolariaceae & Calceolaria dentifolia Edwin & $E$ & & & & & & & $x$ & $x$ & $x$ & \\
\hline Calceolariaceae & Calceolaria linearis Ruiz \& Pav. & $\mathrm{E}$ & & $x$ & & & & & & $x$ & & $x$ \\
\hline Calceolariaceae & Calceolaria rufescens Molau & $E$ & & & & $x$ & & & $x$ & & & \\
\hline Calceolariaceae & Calceolaria sp. & - & & $x$ & & & & & & $x$ & & \\
\hline Calceolariaceae & Calceolaria virgata Ruiz \& Pav. & $\mathrm{N}$ & & & & & & & & $x$ & & \\
\hline Caprifoliaceae & Valeriana punctata F.G. Mey. & $\mathrm{N}$ & & & & & & & & $x$ & & \\
\hline Ephedraceae & Ephedra americana Humb. \& Bonpl. ex Willd. & $\mathrm{N}$ & $x$ & $x$ & & & & & $x$ & & & \\
\hline Ericaceae & Gaultheria bracteata G. Don & $\mathrm{N}$ & & & $x$ & $x$ & $x$ & $x$ & & & & \\
\hline Ericaceae & Gaultheria erecta Vent. & $\mathrm{N}$ & & & & & & & $x$ & $x$ & & $x$ \\
\hline Ericaceae & Pernettya prostrata (Cav.) DC. & $\mathrm{N}$ & & & & & & & $x$ & & $x$ & \\
\hline Ericaceae & Vaccinium cf. crenatum (G. Don) Sleumer & $\mathrm{N}$ & & & & & & & & & $x$ & \\
\hline Fabaceae & Lupinus ramosissimus Benth. & $\mathrm{N}$ & & $x$ & $x$ & & & & & $x$ & & \\
\hline Grossulariaceae & Ribes bolivianum Jancz. & $\mathrm{N}$ & & $x$ & $x$ & $x$ & $x$ & & $x$ & $x$ & & $x$ \\
\hline Hypericaceae & Hypericum laricifolium Juss. & $\mathrm{N}$ & & $x$ & & & & & & & $x$ & \\
\hline Lamiaceae & Clinopodium argenteum (Kunth) Govaerts & $\mathrm{E}$ & & $x$ & & & & & & & & \\
\hline Lamiaceae & Salvia camporum Epling & $\mathrm{E}$ & $x$ & $x$ & & & & & $x$ & $x$ & & \\
\hline Lamiaceae & Salvia corrugata Vahl. & $\mathrm{N}$ & & $x$ & & & & & $x$ & $x$ & & $x$ \\
\hline Lamiaceae & Salvia striata Benth. & $\mathrm{E}$ & & $x$ & $x$ & $x$ & $x$ & & & $x$ & $x$ & \\
\hline Melastomataceae & Brachyotum rostratum (Naudin) Triana & $\mathrm{N}$ & $x$ & & & & & & $x$ & $x$ & & \\
\hline Onagraceae & Fuchsia mathewsii J.F. Macbr. & $\mathrm{E}$ & & & & $x$ & & $x$ & & $x$ & $x$ & $x$ \\
\hline Polygalaceae & Monnina pseudosalicifolia Ferreyra & $\mathrm{N}$ & & & & & & & $x$ & $x$ & $x$ & \\
\hline Polygalaceae & Monnina salicifolia Ruiz \& Pav. & $\mathrm{N}$ & & $x$ & & $x$ & $x$ & & $x$ & $x$ & $x$ & $x$ \\
\hline Polygonaceae & Especie no identificada & - & & & & & & & & & & $x$ \\
\hline Rosaceae & Rubus acanthophyllos Focke & $\mathrm{N}$ & & & & $x$ & $x$ & & & $x$ & $x$ & \\
\hline Solanaceae & Cestrum conglomeratum Ruiz \& Pav. & $\mathrm{N}$ & & & $x$ & & & & & $x$ & & \\
\hline Solanaceae & Solanum ochranthum Dunal & $\mathrm{N}$ & & & & & & & $x$ & $x$ & & \\
\hline
\end{tabular}




\begin{tabular}{|c|c|c|c|c|c|c|c|c|c|c|c|c|}
\hline \multirow{2}{*}{ Familia } & \multirow{2}{*}{ Nombre Científico } & \multirow{2}{*}{ Estatus } & \multicolumn{4}{|c|}{ Pastizales } & \multicolumn{6}{|c|}{ Parches boscosos } \\
\hline & & & L1 & $\mathbf{L 2}$ & L3 & L4 & $\mathbf{L 5}$ & $\mathbf{L 6}$ & L7 & $\mathbf{L 8}$ & L9 & L10 \\
\hline \multicolumn{13}{|l|}{ Subarbustos } \\
\hline Asteraceae & $\begin{array}{l}\text { Ageratina azangaroensis (Sch. Bip. ex Wedd.) R.M. } \\
\text { King \& H. Rob. }\end{array}$ & $\mathrm{N}$ & & $x$ & & & & & & & & $\mathrm{x}$ \\
\hline Asteraceae & Stevia puberula Hook. & $\mathrm{E}$ & & $x$ & $\mathrm{x}$ & & & $x$ & & & & $x$ \\
\hline Asteraceae & Stevia sp. & - & & & & & & & & & $x$ & \\
\hline Melastomataceae & Brachyotum figueroae J.F. Macbr. & $\mathrm{E}$ & & & & & & & & $x$ & & \\
\hline Orobanchaceae & Bartsia crisafullii N.H. Holmgren & $\mathrm{N}$ & & $x$ & $x$ & & $x$ & & & & & \\
\hline Orobanchaceae & Bartsia mutica (Kunth) Benth. & $\mathrm{N}$ & & & & & $x$ & & & & $x$ & \\
\hline Orobanchaceae & Bartsia trichophylla Wedd. & $\mathrm{N}$ & & & & & & & & & & $x$ \\
\hline Scrophulariaceae & Alonsoa linearis (Jacq.) Ruiz \& Pav. & $\mathrm{N}$ & & & & & $x$ & & & $x$ & & \\
\hline Solanaceae & Salpichroa sp. & - & & & & $x$ & & & & & & \\
\hline Solanaceae & Solanum aloysiifolium Dunal & $\mathrm{N}$ & & & & $x$ & & & & & & \\
\hline Urticaceae & Phenax laevigatus Wedd. & $\mathrm{N}$ & & & & $x$ & & & & & & \\
\hline \multicolumn{13}{|l|}{ Matas } \\
\hline Apiaceae & Azorella multifida (Ruiz \& Pav.) Pers. & $\mathrm{N}$ & & $x$ & & $x$ & $x$ & $x$ & $x$ & & & $x$ \\
\hline Asteraceae & Baccharis caespitosa (Ruiz \& Pav.) Pers. & $\mathrm{N}$ & & $x$ & $x$ & $x$ & $x$ & $x$ & & & $x$ & \\
\hline Polygonaceae & Muehlenbeckia volcanica (Benth.) Endl. & $\mathrm{N}$ & $x$ & $x$ & $x$ & & $x$ & $x$ & & & & \\
\hline \multicolumn{13}{|l|}{ Hierbas } \\
\hline \multicolumn{13}{|l|}{$\begin{array}{l}\text { Bulbos (geófi- } \\
\text { tas) }\end{array}$} \\
\hline Amaryllidaceae & Stenomesson miniatum (Herb.) Ravenna & $\mathrm{N}$ & & & & & & & & & & $x$ \\
\hline Asparagaceae & Anthericum sp. & - & & & & & & & & & $x$ & \\
\hline \multicolumn{13}{|c|}{ Hierbas erectas } \\
\hline Alstroemeriaceae & Bomarea dulcis (Hook.) Beauverd & $\mathrm{N}$ & & & $x$ & $x$ & & & & & $\mathrm{x}$ & \\
\hline Apiaceae & Niphogeton dissecta (Benth.) J.F. Macbr. & $\mathrm{N}$ & $x$ & & & $x$ & $x$ & & $\mathrm{x}$ & & & \\
\hline Asteraceae & Achyrocline alata (Kunth) DC. & $\mathrm{N}$ & & $x$ & & $x$ & & & $x$ & $x$ & $x$ & $x$ \\
\hline Asteraceae & Bidens andicola Kunth & $\mathrm{N}$ & $x$ & $x$ & $x$ & $x$ & $x$ & $x$ & & & $x$ & $x$ \\
\hline Asteraceae & Chrysactinium acaule (Kunth) Wedd. & $\mathrm{N}$ & & $x$ & $x$ & & & & & & $x$ & $x$ \\
\hline Asteraceae & Conyza cf. popayanensis (Hieron.) Pruski & $\mathrm{N}$ & & & & & & & & $x$ & & \\
\hline Asteraceae & Conyza primulifolia (Lam.) Cuatrec. \& Lourteig & $\mathrm{N}$ & $x$ & $\mathrm{x}$ & $x$ & & $x$ & $x$ & & & $x$ & $x$ \\
\hline Asteraceae & Conyza sumatrensis (Retz.) E. Walker & I & & $x$ & & $\mathrm{x}$ & & & & & & $x$ \\
\hline Asteraceae & Dendrophorbium chopinii Montesinos & $\mathrm{E}$ & $x$ & $x$ & & $x$ & $x$ & & $\mathrm{x}$ & $x$ & $x$ & $x$ \\
\hline Asteraceae & Dendrophorbium usgorense (Cuatrec.) C. Jeffrey & $\mathrm{E}$ & & & & $x$ & & & $\mathrm{x}$ & & $x$ & \\
\hline Asteraceae & Dorobaea callacallensis (Cuatrec.) B. Nord. \& Pruski & $\mathrm{E}$ & & $x$ & $\mathrm{x}$ & & & $\mathrm{x}$ & & & & \\
\hline Asteraceae & Dorobaea pimpinellifolia (Kunth) B. Nord. & $\mathrm{N}$ & & & $x$ & & & & & & & $x$ \\
\hline Asteraceae & Galinsoga quadriradiata Ruiz \& Pav. & $\mathrm{N}$ & & & & & & & $x$ & & & $x$ \\
\hline Asteraceae & Gamochaeta americana (Mill.) Wedd. & $?$ & $x$ & $\mathrm{x}$ & & & & $x$ & & $x$ & & \\
\hline Asteraceae & Gnaphalium sp. & - & & $x$ & $x$ & $x$ & $x$ & & & & & $x$ \\
\hline Asteraceae & Hieracium mapirense Britton & $\mathrm{N}$ & & $x$ & $x$ & $x$ & & & & & & \\
\hline Asteraceae & Senecio canescens (Bonpl.) Cuatrec. & $\mathrm{N}$ & & & & & & $\mathrm{x}$ & & & & \\
\hline Asteraceae & Senecio coymolachensis Cabrera & $\mathrm{E}$ & & & & & & & & $x$ & & \\
\hline Asteraceae & Sigesbeckia jorullensis Kunth & $\mathrm{N}$ & & & & & & & & $x$ & $x$ & \\
\hline Boraginaceae & Moritzia lindenii (A. DC.) Benth. ex Gürke & $\mathrm{N}$ & & & & & & & $\mathrm{x}$ & & & \\
\hline Brassicaceae & Sisymbrium officinale (L.) Scop. & I & & & & & & & $x$ & & & \\
\hline Campanulaceae & Lobelia tenera Kunth & $\mathrm{N}$ & & $x$ & $x$ & $x$ & & & & $x$ & & $x$ \\
\hline Caprifoliaceae & Valeriana decussata Ruiz \& Pav. & $\mathrm{N}$ & & $x$ & & $x$ & & & & & & \\
\hline Caryophyllaceae & Arenaria lanuginosa (Michx.) Rohrb. & $\mathrm{N}$ & & & & $x$ & & $x$ & & & $x$ & \\
\hline Caryophyllaceae & Cerastium nutans Raf. & I & & & & & & $x$ & & & & \\
\hline Cyperaceae & Carex pichinchensis Kunth & $\mathrm{N}$ & $x$ & $x$ & $x$ & & $x$ & & & $x$ & & \\
\hline Cyperaceae & Oreobolopsis tepalifera T. Koyama \& Guagl. & $\mathrm{N}$ & & $x$ & & & & & & & & \\
\hline Cyperaceae & Rhynchospora macrochaeta Steud. ex Boeckeler & $\mathrm{N}$ & $x$ & & & $\mathrm{x}$ & $x$ & $x$ & & $x$ & $x$ & $x$ \\
\hline Fabaceae & Astragalus garbancillo Cav. & $\mathrm{N}$ & & & $x$ & $x$ & & & & $x$ & $x$ & \\
\hline Fabaceae & Lupinus richardianus C.P. Sm. & $\mathrm{N}$ & & & & & & & & & & $x$ \\
\hline Gentianaceae & Gentianella decemnectaria J.S. Pringle & $\mathrm{E}$ & $x$ & $\mathrm{x}$ & $\mathrm{x}$ & $x$ & & & & & $x$ & $x$ \\
\hline Gentianaceae & Halenia weddelliana Gilg & $\mathrm{N}$ & $x$ & $x$ & $x$ & $x$ & & $\mathrm{x}$ & & $x$ & $x$ & $x$ \\
\hline Hypericaceae & Hypericum silenoides Juss. & $\mathrm{N}$ & & & & & & & & & & $\mathrm{x}$ \\
\hline Iridaceae & Orthrosanthus chimboracensis (Kunth) Baker & $\mathrm{N}$ & $x$ & $x$ & & $\mathrm{x}$ & $x$ & & $\mathrm{x}$ & $x$ & $x$ & $x$ \\
\hline Iridaceae & Sisyrinchium caespitificum Kraenzl. & $\mathrm{N}$ & $x$ & & & & & & & & & \\
\hline Iridaceae & Sisyrinchium cf. iridifolium Kunth & $\mathrm{N}$ & & $x$ & $x$ & $x$ & & & & & & $x$ \\
\hline Juncaceae & Juncus bufonius $\mathrm{L}$. & $\mathrm{I}$ & & & $x$ & & & & & & & \\
\hline
\end{tabular}




\begin{tabular}{|c|c|c|c|c|c|c|c|c|c|c|c|c|}
\hline \multirow{2}{*}{ Familia } & \multirow{2}{*}{ Nombre Científico } & \multirow{2}{*}{ Estatus } & \multicolumn{4}{|c|}{ Pastizales } & \multicolumn{6}{|c|}{ Parches boscosos } \\
\hline & & & L1 & $\mathbf{L 2}$ & L3 & L4 & L5 & L6 & L7 & L8 & L9 & L10 \\
\hline Juncaceae & Luzula racemosa Desv. & $\mathrm{N}$ & $\mathrm{x}$ & $\mathrm{x}$ & $\mathrm{x}$ & $\mathrm{x}$ & & $x$ & & & $\mathrm{x}$ & \\
\hline Lamiaceae & Minthostachys mollis Griseb. & $\mathrm{N}$ & & & $x$ & $x$ & $x$ & & & $x$ & $x$ & \\
\hline Linaceae & Linum prostratum Dombey ex Lam. & $\mathrm{N}$ & & & & & & & & & & $x$ \\
\hline Loasaceae & Nasa basilica T. Henning \& Weigend & $E$ & & & & & & & $x$ & & $x$ & \\
\hline Onagraceae & Epilobium denticulatum Ruiz \& Pav. & $\mathrm{N}$ & & & & & & & & $x$ & & \\
\hline Orchidaceae & Sauroglossum sp. & - & & & & & & & & & & $x$ \\
\hline Orobanchaceae & Castilleja peruviana T.I. Chuang \& Heckard & $E$ & & & & $x$ & & & & & & \\
\hline Orobanchaceae & Castilleja vadosa T.I. Chuang \& Heckard & $\mathrm{E}$ & & $x$ & $x$ & $x$ & & & & & & \\
\hline Orobanchaceae & Lamourouxia sylvatica Kunth & $\mathrm{N}$ & & & & & & & & & & $x$ \\
\hline Polygonaceae & Rumex peruanus Rech. F & $\mathrm{N}$ & & $x$ & & $x$ & & & $x$ & $x$ & & \\
\hline Ranunculaceae & Ranunculus peruvianus Pers. & $\mathrm{N}$ & & $x$ & $x$ & $x$ & & $x$ & & & & \\
\hline Rosaceae & Geum peruvianum Focke & $\mathrm{N}$ & & & & $x$ & & & & & & \\
\hline Rubiaceae & Colignonia parviflora (Kunth) Choisy & $\mathrm{N}$ & & & & & & & $x$ & $x$ & $x$ & \\
\hline Scrophulariaceae & Alonsoa minor Edwin & $E$ & & & & & & $x$ & & & & \\
\hline Solanaceae & Solanum sp. & - & & $x$ & & $x$ & & $x$ & $x$ & $x$ & $x$ & \\
\hline Urticaceae & Urtica leptophylla Kunth & $\mathrm{N}$ & $x$ & $x$ & $x$ & & & $x$ & $x$ & $x$ & $x$ & \\
\hline Verbenaceae & Verbena litoralis Kunth & $\mathrm{N}$ & & $x$ & & $x$ & & & & & & \\
\hline \multicolumn{13}{|c|}{$\begin{array}{l}\text { Hierbas postra- } \\
\text { das }\end{array}$} \\
\hline Amaranthaceae & Alternanthera macbridei Standl. & $\mathrm{N}$ & & & $x$ & $x$ & $x$ & & & & & $x$ \\
\hline Apiaceae & $\begin{array}{l}\text { Cyclospermum leptophyllum (Pers.) Sprague ex Britton } \\
\text { \& P. Wilson }\end{array}$ & $?$ & $x$ & & & & & & & & $x$ & \\
\hline Apiaceae & Hydrocotyle incrassata Ruiz \& Pav. & $\mathrm{N}$ & & $x$ & $x$ & & & $x$ & & & & $x$ \\
\hline Apiaceae & Oreomyrrhis andicola (Kunth) Endl. ex Hook. f. & $\mathrm{N}$ & $x$ & $x$ & $x$ & $x$ & $x$ & $x$ & & & $x$ & $x$ \\
\hline Asteraceae & Cotula mexicana (DC.) Cabrera & $\mathrm{N}$ & $x$ & $x$ & $x$ & & & & & & & \\
\hline Asteraceae & Heliopsis buphthalmoides (Jacq.) Dunal & $\mathrm{N}$ & & & & & & & & $x$ & & \\
\hline Asteraceae & Oritrophium hirtopilosum (Hieron.) Cuatrec. & $\mathrm{E}$ & & & $x$ & $x$ & & & & $x$ & & $x$ \\
\hline Boraginaceae & Lithospermum peruvianum A. DC. & $\mathrm{N}$ & & & $x$ & $x$ & & & & & $x$ & $x$ \\
\hline Boraginaceae & Plagiobothrys sp. & - & & & & & & & & $x$ & & \\
\hline Brassicaceae & Lepidium bipinnatifidum Desv. & $\mathrm{N}$ & & & & & & & & $x$ & & \\
\hline Brassicaceae & Nasturtium officinale W.T. Aiton & I & & & & & & $x$ & & $x$ & & \\
\hline Campanulaceae & Wahlenbergia peruviana A. Gray & $\mathrm{N}$ & & & & $x$ & & & & & & \\
\hline Caprifoliaceae & Valeriana plantaginea Kunth & $\mathrm{N}$ & & $\mathrm{x}$ & $x$ & $x$ & $x$ & & & $x$ & $x$ & $x$ \\
\hline Caryophyllaceae & Arenaria aphanantha Wedd. & $\mathrm{N}$ & & & $x$ & & & & & & $x$ & \\
\hline Caryophyllaceae & Arenaria musciformis Triana \& Planch. & $\mathrm{N}$ & $x$ & & & & & $x$ & & & $x$ & \\
\hline Caryophyllaceae & Arenaria sp. & - & $x$ & & & & & & & & & \\
\hline Caryophyllaceae & Cerastium sp. 1 & - & $x$ & $x$ & $x$ & & & $x$ & & $x$ & $x$ & \\
\hline Caryophyllaceae & Cerastium sp. 2 & - & & & & $x$ & & & & & $x$ & \\
\hline Caryophyllaceae & Drymaria cordata (L.) Willd. ex Schult. & $\mathrm{N}$ & & & & $x$ & & & & & $x$ & \\
\hline Caryophyllaceae & Drymaria glaberrima Bartl. & $E$ & & & & & & & & $x$ & & \\
\hline Caryophyllaceae & Paronychia macbridei Chaudhri & $E$ & & & $x$ & & & & $x$ & & & \\
\hline Caryophyllaceae & Silene thysanodes Fenzl & $\mathrm{N}$ & & & $x$ & $x$ & & & & & $x$ & $x$ \\
\hline Caryophyllaceae & Stellaria cuspidata Willd. ex D.F.K. Schltdl. & $\mathrm{N}$ & $x$ & & & & & $x$ & $x$ & $x$ & $x$ & \\
\hline Fabaceae & Lupinus peruvianus Ulbr. & $\mathrm{E}$ & $x$ & $x$ & & $x$ & & $x$ & & & $x$ & \\
\hline Fabaceae & Trifolium amabile Kunth & $\mathrm{N}$ & $x$ & $x$ & & $x$ & & $x$ & & $x$ & $x$ & \\
\hline Gentianaceae & Gentiana sedifolia Kunth & $\mathrm{N}$ & & $x$ & & & & $x$ & & & & \\
\hline Geraniaceae & Geranium ayavacense Willd. ex Kunth & $\mathrm{N}$ & $x$ & $x$ & $x$ & $x$ & & $x$ & & & $x$ & $x$ \\
\hline Geraniaceae & Geranium chilloense Willd. ex Kunth & $\mathrm{N}$ & & & & & & & & & $x$ & \\
\hline Geraniaceae & Geranium multipartitum Benth. & $\mathrm{N}$ & & $x$ & & & & & & & & \\
\hline Geraniaceae & Geranium peruvianum Hieron. & $\mathrm{E}$ & $x$ & & $x$ & $x$ & $x$ & $x$ & $x$ & & $x$ & $x$ \\
\hline Gunneraceae & Gunnera magellanica Lam. & $\mathrm{N}$ & $x$ & & & & & & & & & \\
\hline Melastomataceae & Miconia chionophila Naudin & $\mathrm{N}$ & & $x$ & $x$ & & & $x$ & $x$ & $x$ & & \\
\hline Onagraceae & Oenothera epilobiifolia Kunth & $\mathrm{N}$ & $x$ & & $x$ & $x$ & $x$ & $x$ & & & $x$ & \\
\hline Orobanchaceae & Castilleja arvensis Schltdl. \& Cham. & $\mathrm{N}$ & & $x$ & $x$ & $x$ & & & & & & \\
\hline Oxalidaceae & Oxalis medicaginea Kunth & $\mathrm{N}$ & & & & $x$ & & & & $x$ & $x$ & \\
\hline Plantaginaceae & Veronica serpyllifolia L. & $\mathrm{I}$ & $x$ & & & & $x$ & & & $x$ & $x$ & \\
\hline Ranunculaceae & Ranunculus praemorsus Kunth ex DC. & $\mathrm{N}$ & $x$ & $x$ & $x$ & $x$ & & $x$ & & & $x$ & \\
\hline Rosaceae & Acaena ovalifolia Ruiz \& Pav. & $\mathrm{N}$ & $x$ & $x$ & $x$ & $x$ & $x$ & $x$ & & $x$ & $x$ & $x$ \\
\hline Rosaceae & Lachemilla aphanoides (Mutis ex L. f.) Rothm. & $\mathrm{N}$ & & $x$ & & & & & & & $x$ & \\
\hline Rosaceae & Lachemilla cf. andina (L.M. Perry) Rothm. & $\mathrm{N}$ & & & & & & & & & & $x$ \\
\hline
\end{tabular}




\begin{tabular}{|c|c|c|c|c|c|c|c|c|c|c|c|c|}
\hline \multirow{2}{*}{ Familia } & \multirow{2}{*}{ Nombre Científico } & \multirow{2}{*}{ Estatus } & \multicolumn{4}{|c|}{ Pastizales } & \multicolumn{6}{|c|}{ Parches boscosos } \\
\hline & & & L1 & $\mathbf{L 2}$ & L3 & L4 & $\mathbf{L 5}$ & L6 & L7 & $\mathbf{L 8}$ & L9 & L10 \\
\hline Rosaceae & Lachemilla orbiculata (Ruiz \& Pav.) Rydb. & $\mathrm{N}$ & $\mathrm{x}$ & $\mathrm{x}$ & $\mathrm{x}$ & $\mathrm{x}$ & $x$ & $\mathrm{x}$ & & $\mathrm{x}$ & $x$ & \\
\hline Rosaceae & Lachemilla pinnata (Ruiz \& Pav.) Rothm. & $\mathrm{N}$ & & $x$ & $x$ & $x$ & & $x$ & & & $x$ & \\
\hline Rubiaceae & Galium aparine $\mathrm{L}$. & I & & & & $x$ & $x$ & $x$ & & & $x$ & $x$ \\
\hline Rubiaceae & Galium canescens Kunth & $\mathrm{N}$ & $x$ & & $x$ & $x$ & & & & & $x$ & \\
\hline Rubiaceae & Galium corymbosum Ruiz \& Pav. & $\mathrm{N}$ & & & $x$ & & & & & $x$ & & \\
\hline Rubiaceae & Galium ferrugineum K. Krause & $\mathrm{N}$ & & $x$ & & & & & & $x$ & $x$ & \\
\hline Rubiaceae & Galium hypocarpium (L.) Endl. ex Griseb. & $\mathrm{N}$ & & & & & & & & $x$ & & \\
\hline Urticaceae & Pilea dombeyana Wedd. & $E$ & $x$ & & & & & & & & & \\
\hline Urticaceae & Pilea microphylla (L.) Liebm. & $\mathrm{N}$ & & & & & & & & & $x$ & \\
\hline Verbenaceae & Junellia fasciculata (Benth.) N. O'Leary \& P. Peralta & $\mathrm{N}$ & $x$ & & $x$ & $x$ & $x$ & $x$ & $x$ & & $x$ & $x$ \\
\hline Verbenaceae & Stachys peruviana Dombey ex Benth. & $E$ & $x$ & $x$ & $x$ & $x$ & & $x$ & & & & $x$ \\
\hline \multicolumn{13}{|l|}{$\begin{array}{l}\text { Hierbas } \\
\text { arrosetadas }\end{array}$} \\
\hline Apiaceae & Azorella biloba (Schltdl.) Wedd. & $\mathrm{N}$ & & & $x$ & & & & & & & \\
\hline Apiaceae & Eryngium humile Cav. & $\mathrm{N}$ & & $\mathrm{x}$ & $x$ & & & & & & & $x$ \\
\hline Asteraceae & Aphanactis villosa S.F. Blake & $\mathrm{N}$ & & $x$ & $x$ & & & $x$ & & & & \\
\hline Asteraceae & Belloa sp. & - & & $x$ & & & & & & & & $x$ \\
\hline Asteraceae & Chevreulia acuminata Less. & $\mathrm{N}$ & & & $x$ & & & & & & & \\
\hline Asteraceae & Hypochaeris sessiliflora Kunth & $\mathrm{N}$ & & $x$ & $x$ & $x$ & & $x$ & & & & \\
\hline Asteraceae & Paranephelius uniflorus Poepp. & $\mathrm{N}$ & & $x$ & $x$ & $x$ & & $x$ & & & & \\
\hline Asteraceae & Perezia pungens (Bonpl.) Less. & $\mathrm{N}$ & & $x$ & & $x$ & & & & & & $x$ \\
\hline Asteraceae & $\begin{array}{l}\text { Senecio repens Stokes var. macbridei (Cuatrec.) } \\
\text { Cabrera }\end{array}$ & $\mathrm{E}$ & & $x$ & & & & & & & & \\
\hline Asteraceae & Taraxacum officinale L. & I & $x$ & $x$ & $x$ & $x$ & & & & & $x$ & \\
\hline Asteraceae & Werneria nubigena Kunth & $\mathrm{N}$ & & $x$ & & & & & & & & \\
\hline Caprifoliaceae & Phyllactis sp. & - & & $x$ & & & & $x$ & & & & \\
\hline Plantaginaceae & Plantago linearis Kunth & $\mathrm{N}$ & $x$ & $x$ & & $x$ & $x$ & $x$ & & & $x$ & \\
\hline Plantaginaceae & Plantago major L. & I & & & $x$ & & & & $x$ & & & $x$ \\
\hline \multicolumn{13}{|c|}{ Hierbas epífitas } \\
\hline Araceae & Anthurium sp. & - & & & & & & & $x$ & & & \\
\hline Bromeliaceae & Tillandsia cf. ferreyrae L.B. Sm. & $\mathrm{E}$ & & & & & & & & $x$ & & \\
\hline Bromeliaceae & Tillandsia sp. & - & & & & & & & & & $x$ & \\
\hline Orchidaceae & Epidendrum sp. & - & & & & & & & & $x$ & & \\
\hline Orchidaceae & Stelis parviflora (Ruiz \& Pav.) Pers. & $E$ & & & & & & & & $x$ & & \\
\hline \multicolumn{13}{|l|}{ Trepadoras } \\
\hline Alstroemeriaceae & Bomarea goniocaulon Baker & $E$ & & & & & & & $x$ & $x$ & & \\
\hline Apiaceae & Arracacia elata $\mathrm{H}$. Wolff & $\mathrm{N}$ & & & $x$ & & & & & $x$ & & \\
\hline Apiaceae & Bowlesia lobata Ruiz \& Pav. & $\mathrm{N}$ & $x$ & & $x$ & & & $x$ & & & $x$ & \\
\hline Apocynaceae & Ditassa weberbaueri Schltr. & $\mathrm{N}$ & $x$ & $x$ & $x$ & $x$ & & & & & $x$ & \\
\hline Apocynaceae & Metastelma sp. & - & & & & $x$ & & & & & & \\
\hline Asteraceae & Jungia rugosa Less. & $\mathrm{N}$ & & $x$ & & & & & & & $x$ & \\
\hline Asteraceae & Lasiocephalus sp. & - & & & & & & & & $x$ & $x$ & $x$ \\
\hline Asteraceae & Munnozia lyrata (A. Gray) H. Rob. \& Brettell & $\mathrm{E}$ & & & & & & $x$ & $x$ & & $x$ & \\
\hline Basellaceae & Ullucus tuberosus Caldas & $\mathrm{N}$ & & & & & & & $x$ & & & \\
\hline Caprifoliaceae & Valeriana clematitis Kunth & $\mathrm{N}$ & & & & & & $x$ & $x$ & $x$ & $x$ & \\
\hline Caryophyllaceae & Drymaria grandiflora Bartl. & $\mathrm{E}$ & & & & & & & $x$ & $x$ & & \\
\hline Cleomaceae & Cleome lechleri Eichler & $\mathrm{N}$ & & & & $x$ & & & & $x$ & $x$ & \\
\hline Fabaceae & Lathyrus magellanicus Lam. & $\mathrm{N}$ & & $x$ & & & & $x$ & & & & \\
\hline Fabaceae & Vicia andicola Kunth & $\mathrm{N}$ & $x$ & $x$ & $x$ & $x$ & & $x$ & & $x$ & $x$ & $x$ \\
\hline Fabaceae & Vicia graminea Sm. & $\mathrm{N}$ & & $x$ & $x$ & & & $x$ & & & & \\
\hline Oxalidaceae & Oxalis Iucumayensis R. Knuth & $\mathrm{N}$ & & & & & & & $x$ & & $x$ & \\
\hline Passifloraceae & Passiflora sp. & - & & & & $x$ & $x$ & & & & $x$ & \\
\hline Ranunculaceae & Thalictrum decipiens B. Boivin & $\mathrm{N}$ & & & & & & & & & & $x$ \\
\hline \multicolumn{13}{|l|}{$\begin{array}{l}\text { Plantas crasas } \\
\text { (Suculentas) }\end{array}$} \\
\hline Crassulaceae & Echeveria sp & - & & & & & & & $x$ & $x$ & & \\
\hline Crassulaceae & Sedum reniforme (H. Jacobsen) Thiede \& 't Hart & $\mathrm{E}$ & & & $x$ & $x$ & & & & & & \\
\hline Piperaceae & Peperomia andina Pino & $E$ & & & & & & $x$ & & & & \\
\hline Piperaceae & Peperomia crystallina Ruiz \& Pav. & $E$ & & & & & & & & & $x$ & \\
\hline Piperaceae & Peperomia hartwegiana Miq. & $\mathrm{N}$ & & $x$ & $\mathrm{x}$ & $x$ & $x$ & & & $x$ & $x$ & \\
\hline
\end{tabular}




\begin{tabular}{|c|c|c|c|c|c|c|c|c|c|c|c|c|}
\hline \multirow{2}{*}{ Familia } & \multirow{2}{*}{ Nombre Científico } & \multirow{2}{*}{ Estatus } & \multicolumn{4}{|c|}{ Pastizales } & \multicolumn{6}{|c|}{ Parches boscosos } \\
\hline & & & $\mathbf{L 1}$ & $\mathbf{L} 2$ & L3 & $\mathbf{L 4}$ & $\mathbf{L 5}$ & $\mathbf{L 6}$ & L7 & $\mathbf{L 8}$ & L9 & L10 \\
\hline Piperaceae & Peperomia rotundata Kunth & $\mathrm{N}$ & $\mathrm{x}$ & & $\mathrm{x}$ & $\mathrm{x}$ & & & $\mathrm{x}$ & & $\mathrm{x}$ & \\
\hline Piperaceae & Peperomia sp. 1 & - & & & & $x$ & & & & & & \\
\hline Piperaceae & Peperomia sp. 2 & - & & & & $x$ & & & & & $x$ & \\
\hline \multicolumn{13}{|l|}{ Gramíneas } \\
\hline Poaceae & Agrostis breviculmis Hitchc. & $\mathrm{N}$ & $x$ & $x$ & & & & & & & $x$ & \\
\hline Poaceae & Agrostis sp. & - & & & & & & & & & & $x$ \\
\hline Poaceae & Agrostis tolucensis Kunth & $\mathrm{N}$ & & $x$ & $x$ & $x$ & & $\mathrm{x}$ & & & $x$ & \\
\hline Poaceae & Bromus catharticus Vahl & $\mathrm{N}$ & & & & $x$ & & & & & & \\
\hline Poaceae & Bromus lanatus Kunth & $\mathrm{N}$ & $x$ & $x$ & $x$ & $x$ & & & & & $x$ & \\
\hline Poaceae & Calamagrostis ampliflora Tovar & $\mathrm{N}$ & & & & & & & & $x$ & $x$ & \\
\hline Poaceae & Calamagrostis intermedia (J. Presl) Steud. & $\mathrm{N}$ & & & & $\mathrm{x}$ & & & & & $x$ & \\
\hline Poaceae & Calamagrostis recta (Kunth) Trin. ex Steud. & $\mathrm{N}$ & & & $\mathrm{x}$ & & & & & & & $x$ \\
\hline Poaceae & Calamagrostis rigida (Kunth) Trin. ex Steud. & $\mathrm{N}$ & $\mathrm{x}$ & $\mathrm{x}$ & $x$ & $\mathrm{x}$ & & $\mathrm{x}$ & & $x$ & $x$ & $\mathrm{x}$ \\
\hline Poaceae & Cortaderia bifida Pilg. & $\mathrm{N}$ & & $x$ & & $x$ & & & & $x$ & & \\
\hline Poaceae & Deyeuxia rigescens (J. Presl) Türpe & $\mathrm{N}$ & & $\mathrm{x}$ & & & & & & & & \\
\hline Poaceae & Deyeuxia tarmensis (Pilg.) Sodiro & $\mathrm{N}$ & & $x$ & $\mathrm{x}$ & & & & $\mathrm{x}$ & & $x$ & \\
\hline Poaceae & Elymus angulatus J. Presl & $\mathrm{N}$ & & & & & & & & $\mathrm{x}$ & & \\
\hline Poaceae & Festuca glabrata Tovar & $\mathrm{E}$ & $\mathrm{x}$ & $\mathrm{x}$ & $\mathrm{x}$ & $\mathrm{x}$ & & $x$ & $\mathrm{x}$ & $x$ & $x$ & $x$ \\
\hline Poaceae & Holcus lanatus L. & I & $x$ & & & & & $x$ & & & & \\
\hline Poaceae & Paspalum bonplandianum Flüggé & $\mathrm{N}$ & $x$ & $x$ & $x$ & $x$ & $x$ & $x$ & & & $x$ & $x$ \\
\hline Poaceae & Paspalum pilgerianum Chase & $\mathrm{N}$ & $x$ & & & & & & & $x$ & $x$ & \\
\hline Poaceae & Trisetum spicatum (L.) K. Richt. & $?$ & $x$ & & $x$ & & & $x$ & & & & $x$ \\
\hline Poaceae & Vulpia myuros (L.) C.C. Gmel. & I & & & & & & & & & $x$ & \\
\hline \multicolumn{13}{|l|}{ Helechos } \\
\hline Aspleniaceae & Asplenium castaneum Schltdl. \& Cham. & $\mathrm{N}$ & & & $\mathrm{x}$ & & & $x$ & & & & \\
\hline Aspleniaceae & Asplenium extensum Fée & $\mathrm{N}$ & & & & & $x$ & & $x$ & & $x$ & \\
\hline Aspleniaceae & Asplenium monanthes $\mathrm{L}$. & $\mathrm{N}$ & & $\mathrm{x}$ & & $\mathrm{x}$ & $x$ & $x$ & & & $x$ & \\
\hline Blechnaceae & Blechnum loxense (Kunth) Hook. ex Salomon & $\mathrm{N}$ & & $\mathrm{x}$ & & & & & & $\mathrm{x}$ & & \\
\hline Dryopteridaceae & Cystopteris fragilis (L.) Bernh. & I & $x$ & $x$ & & & & $x$ & $\mathrm{x}$ & & $x$ & \\
\hline Dryopteridaceae & Dryopteris sp. & - & $x$ & $x$ & & $\mathrm{x}$ & $\mathrm{x}$ & & & $x$ & $x$ & \\
\hline Dryopteridaceae & Dryopteris wallichiana (Spreng.) Hyl. & I & & $x$ & & & & & $x$ & $x$ & & \\
\hline Dryopteridaceae & Megalastrum sp. & - & & & & & & & & $x$ & & \\
\hline Dryopteridaceae & Polystichum cochleatum (Klotzsch) Hieron. & $\mathrm{N}$ & $\mathrm{x}$ & & $\mathrm{x}$ & $x$ & $x$ & $x$ & $x$ & & $\mathrm{x}$ & \\
\hline Equisetaceae & Equisetum bogotense Kunth & $\mathrm{N}$ & & & & & & & & $x$ & $x$ & \\
\hline Gleicheniaceae & Dicranopteris flexuosa (Schrad.) Underw. & $\mathrm{N}$ & & & & & & & & $x$ & & \\
\hline Lycopodiaceae & Lycopodium clavatum L. & $?$ & & $\mathrm{x}$ & & & & & & $x$ & & $x$ \\
\hline Lycopodiaceae & Phlegmariurus hartwegianus (Spring) B. Øllg. & $\mathrm{N}$ & & $\mathrm{x}$ & & & & & & & & \\
\hline Polypodiaceae & Campyloneurum amphostenon (Kunze ex Klotzsch) Fée & $\mathrm{N}$ & & $x$ & & & $x$ & & & $\mathrm{x}$ & $\mathrm{x}$ & $x$ \\
\hline Polypodiaceae & $\begin{array}{l}\text { Campyloneurum angustipaleatum (Alston) M. Mey. ex } \\
\text { Lellinger }\end{array}$ & $\mathrm{N}$ & & & & $\mathrm{x}$ & & & & $x$ & & \\
\hline Polypodiaceae & Campyloneurum densifolium (Hieron.) Lellinger & $\mathrm{N}$ & & & & & & & $\mathrm{x}$ & $\mathrm{x}$ & & \\
\hline Polypodiaceae & Elaphoglossum engelii (H. Karst.) Christ & $\mathrm{N}$ & $\mathrm{x}$ & $\mathrm{x}$ & $\mathrm{x}$ & $\mathrm{x}$ & & $\mathrm{x}$ & $\mathrm{x}$ & $x$ & $\mathrm{x}$ & $\mathrm{x}$ \\
\hline Polypodiaceae & Elaphoglossum minutum (Pohl ex Fée) T. Moore & $\mathrm{N}$ & & & & $x$ & & & & & $x$ & \\
\hline Polypodiaceae & Elaphoglossum rosenstockii Christ ex Rosenst. & $\mathrm{N}$ & & $x$ & & $\mathrm{x}$ & $\mathrm{x}$ & & $\mathrm{x}$ & & $\mathrm{x}$ & $\mathrm{x}$ \\
\hline Polypodiaceae & $\begin{array}{l}\text { Melpomene moniliformis (Lag. ex Sw.) A.R. Sm. \& R.C. } \\
\text { Moran }\end{array}$ & $\mathrm{N}$ & & & & & & & & $\mathrm{x}$ & & \\
\hline Polypodiaceae & Nephrolepis sp. & - & & & & & & & & $\mathrm{x}$ & & \\
\hline Polypodiaceae & Pecluma curvans (Mett.) M.G. Price & $\mathrm{N}$ & & & & & & & & & $\mathrm{x}$ & \\
\hline Polypodiaceae & Pleopeltis buchtienii (Christ \& Rosenst.) A.R. Sm. & $\mathrm{N}$ & & $\mathrm{x}$ & & & & & & $\mathrm{x}$ & $\mathrm{x}$ & \\
\hline Polypodiaceae & Polypodium chrysolepis Hook. & $\mathrm{N}$ & & & $x$ & & & & & $x$ & & \\
\hline Polypodiaceae & Polypodium pycnocarpum C. Chr. & $\mathrm{N}$ & & $\mathrm{x}$ & $x$ & $\mathrm{x}$ & $x$ & & & & $\mathrm{x}$ & \\
\hline Pteridaceae & Adiantum sp. & - & & & & & & & $\mathrm{x}$ & & $x$ & $\mathrm{x}$ \\
\hline Pteridaceae & Cheilanthes cf. peruviana (Desv.) T. Moore & $E$ & & & & & & & & $x$ & $x$ & \\
\hline Pteridaceae & Jamesonia alstonii A.F. Tryon & $\mathrm{N}$ & $x$ & & & & & & & & & \\
\hline Selaginellaceae & Selaginella ramosissima Baker & $\mathrm{N}$ & & $\mathrm{x}$ & & & & & & & & $x$ \\
\hline Thelypteridaceae & Thelypteris rufa (Poir.) A.R. Sm. & $\mathrm{N}$ & & & & & & & $\mathrm{x}$ & $x$ & $x$ & \\
\hline
\end{tabular}




\section{RESULTADOS}

\section{Composición y diversidad de especies}

Del total de 294 especies, divididas en 190 géneros y 65 familias (Tabla 2), tanto en pajonales como en zonas boscosas las familias más diversas registradas fueron: Asteraceae (44 géneros, 74 especies), Poaceae (12 géneros, 19 especies), Caryophyllaceae (6 géneros, 13 especies), Polypodiaceae (7 géneros, 12 especies), Rosaceae (6 géneros, 10 especies), Apiaceae ( 8 géneros, 9 especies), Fabaceae ( 6 géneros, 9 especies), Calceolariaceae (1 género, 8 especies), Orobanchaceae (3 géneros, 7 especies), Solanaceae ( 3 géneros, 7 especies), Piperaceae ( 1 género, 6 especies), Rubiaceae ( 2 géneros, 6 especies), Caprifoliaceae ( 2 géneros, 5 especies), Dryopteridaceae (4 géneros, 5 especies), Lamiaceae (3 géneros, 5 especies). En Pteridophyta, se registraron 10 familias, 22 géneros y 30 especies. Los géneros con mayor riqueza de especies fueron: Baccharis (Asteaceae) con 9 especies, Senecio (Asteraceae), 9 especies, Calceolaria (Calceolariaceae) con 7 especies, Peperomia (Piperaceae) con 6 especies, Galium (Rubiaceae) con 5 especies, Solanum (Solanaceae) con 5 especies, Arenaria (Caryophyllaceae), Calamagrostis (Poaceae), Geranium (Geraniaceae), Gynoxys (Asteraceae), Lachemilla (Rosaceae) y Valeriana (Caprifoliaceae) con 4 especies cada una.

En los pajonales se registraron 356 ocurrencias representadas por 185 especies, pertenecientes a 130 géneros y 39 familias. Del total de especies registradas, el 1,6\% (3) fueron árboles, el $17,8 \%$ (33) fueron arbustos, el 2,2\% (4) fueron subarbustos, el 1,6\% (3) fueron matas arbustivas, el 50,2\% (93) fueron hierbas (bulbosas, erectas, postradas o en rosetas), el 5,4\% (10) fueron trepadoras, el 10,3\% (19) fueron helechos, el $8,1 \%$ (15) fueron gramíneas y el 3\% (5) fueron plantas crasas. En zonas boscosas se registraron 491 ocurrencias categorizadas en 264 especies, y pertenecientes a 174 géneros y 64 familias. Del total de especies halladas, el $6,8 \%$ (18) fueron árboles, el 22,3\% (59) fueron arbustos, el 3,8\% (10) fueron subarbustos, el $1,1 \%$ (3) fueron matas arbustivas, el $40,2 \%$ (106) fueron hierbas (bulbosas, erectas, postradas, en rosetas o epífitas), el 6,4\% (17) fueron trepadoras, el $10,6 \%$ (28) fueron helechos, el $6,4 \%$ (17) fueron gramíneas y el $2 \%$ (6) fueron plantas crasas. Por otro lado, en pajonales, de las 185 especies de origen conocido, el $10,3 \%$ (19 especies) fueron exóticas, mientras que, en zonas boscosas, de las 264 especies conocidas, el 8,3\% (22 especies) son exóticas. Tanto en los pajonales como en zonas boscosas, las especies con mayor representatividad (ocurrencia) son: Festuca glabrata Tovar (Poaceae), Elaphoglossum engelii (H. Karst.) Christ (Polypodiaceae), Acaena ovalifolia Ruiz \& Pav. (Rosaceae), Oreomyrrhis andicola (Kunth) Endl. ex Hook. f. (Apiaceae), Bidens andicola Kunth (Asteraceae), Dendrophorbium chopinii Montesinos (Asteraceae), Vicia andicola Kunth (Fabaceae), Halenia weddelliana Gilg (Gentianaceae), Geranium peruvianum Hieron. (Genraniaceae), Orthrosanthus chimboracensis (Kunth) Baker (Iridaceae), Calamagrostis rigida (Kunth) Trin. ex Steud. (Poaceae), Paspalum bonplandianum Flüggé (Poaceae), Junellia fasciculata (Benth.) N. O'Leary \& P. Peralta (Verbenaceae) y Lachemilla orbiculata (Ruiz \& Pav.) Rydb. (Rosaceae).

Las zonas boscosas están representadas por las siguientes 19 especies arbóreas u leñosas: Sambucus peruviana Kunth (Adoxaceae), Gynoxys caracensis Muschl., Gynoxys cerrateana B. Herrera, Gynoxys soukupii Cuatrec., Gynoxys sp., Jungia schuerae Harling, Pentacalia peruviana (Pers.) Cuatrec., Verbesina cf. ochroleucotricha Sagást., Verbesina cumingii (Griseb.) S.F. Blake (Asteraceae), Siphocampylus matthiaei A. DC. (Campanulaceae), Vallea stipularis L. f. (Elaeocarpaceae), Escallonia sp. (Escalloniaceae), Otholobium mexicanum (L. f.) J.W. Grimes (Fabaceae), Buddleja incana Ruiz \& Pav. (Loganiaceae), Hesperomeles cuneata Lindl, Hesperomeles ferruginea (Pers.) Benth., Polylepis multijuga Pilg. (Rosaceae), Solanum barbulatum Zahlbr., Solanum sanchez-vegaeS. Knapp (Solanaceae). 


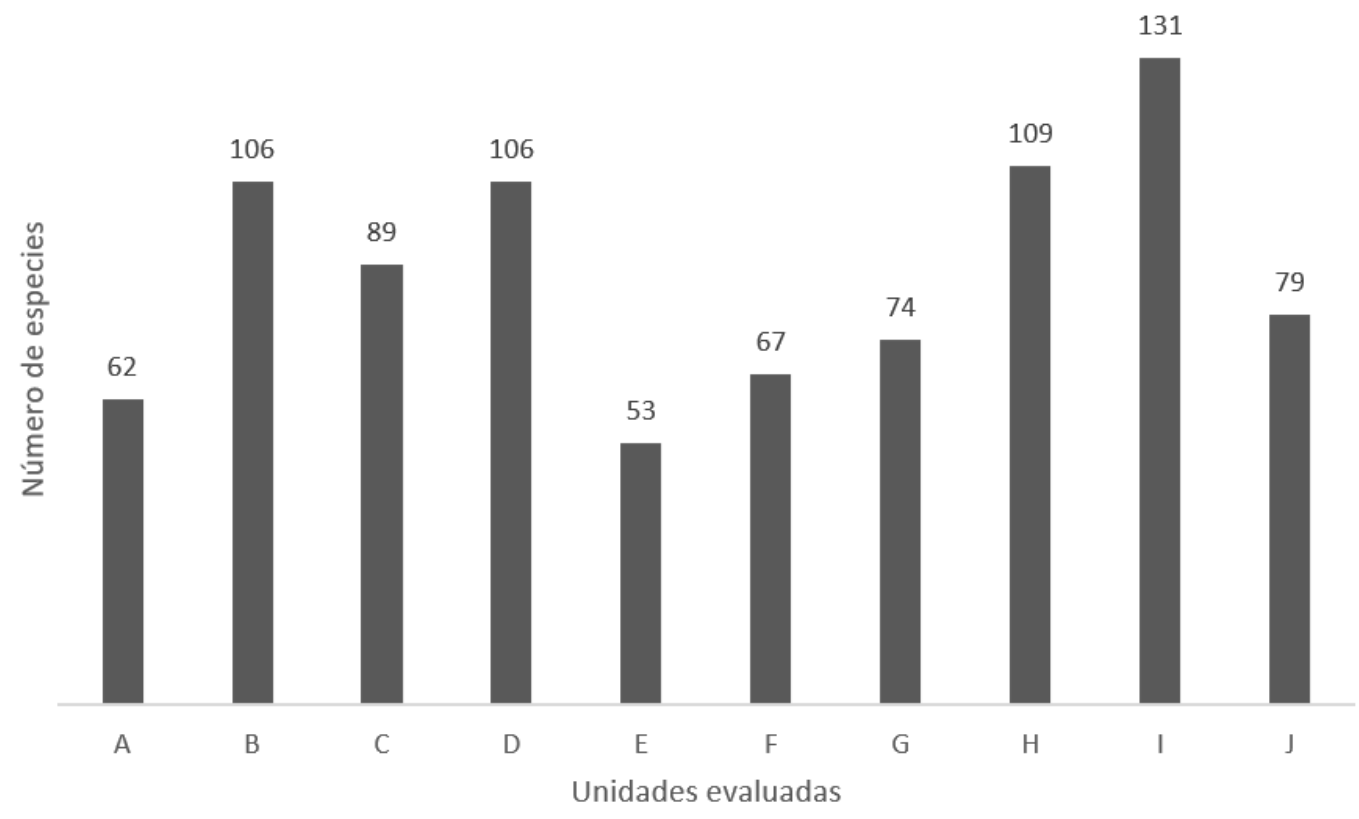

Figura 2. Número total de especies obtenido de acuerdo al área evaluada. A) Pajonales en ruinas arqueológicas enterradas, B) Pajonales con influencia de fuego, C) Cima de montaña, D) Vegetación en ruinas arqueológicas expuestas, E) Parche boscoso en zona arqueológica con reciente abandono, F) Vegetación en hundimientos kársticos, G) Vegetación en hundimiento kárstico profundo, H) Vegetación de quebradas ocasionalmente secas, I) Vegetación en ruinas arqueológicas con densa cobertura arbórea y J) Vegetación arbustiva en zona de riscos.

\section{Comparación entre los pajonales y zonas boscosas}

En la Figura 2 se ilustra el número total de especies obtenido según el área evaluada. Las unidades A-D representa los pajonales y donde $B$ y $D$ que representan tanto a zonas con influencia de fuego (pajonales en andenería enterrada) y vegetación en ruinas arqueológicas expuestas presentan alta diversidad con 106 especies cada una siendo relativamente menor en $C$ y $A$. Las zonas boscosas corresponden a $E, F, G, H$, I y $\mathrm{J}$ donde la unidad en ruinas arqueológicas con densa cobertura arbórea presenta el mayor número de especies con 131, seguido de la unidad de vegetación en quebradas ocasionalmente secas con 109 especies, mientras que el parche boscoso en zona arqueológica con reciente abandono, vegetación en hundimientos kársticos y en elemento kárstico profundo presentan 53, 67 y 74 especies respectivamente. Respecto a especies exóticas, las unidades correspondientes a vegetación en hundimientos kársticos y en ruinas arqueológicas con densa cobertura arbórea presentan 5 especies cada una mientras que el parche boscoso en zona arqueológica con reciente abandono presenta únicamente 2 especies introducidas.

La diversidad de flora puede estar relacionada a la mayor humedad en las depresiones de origen kársticos y los parches boscosos no intervenidos, evidenciando la particularidad de la vegetación de las zonas boscosas en composición, diversidad y cobertura, en comparación con los pajonales.

\section{Endemismo en el complejo arqueológico de La Boveda}

Siguiendo las referencias de León et al. (2006), Montesinos-Tubée (2017a) y Tropicos (2020) para la definición de endemismos, del total de 294 especies halladas en el estudio, 56 especies son endémicas correspondiendo al $19 \%$ de la flora registrada (Tabla 1). Las familias con ma- 


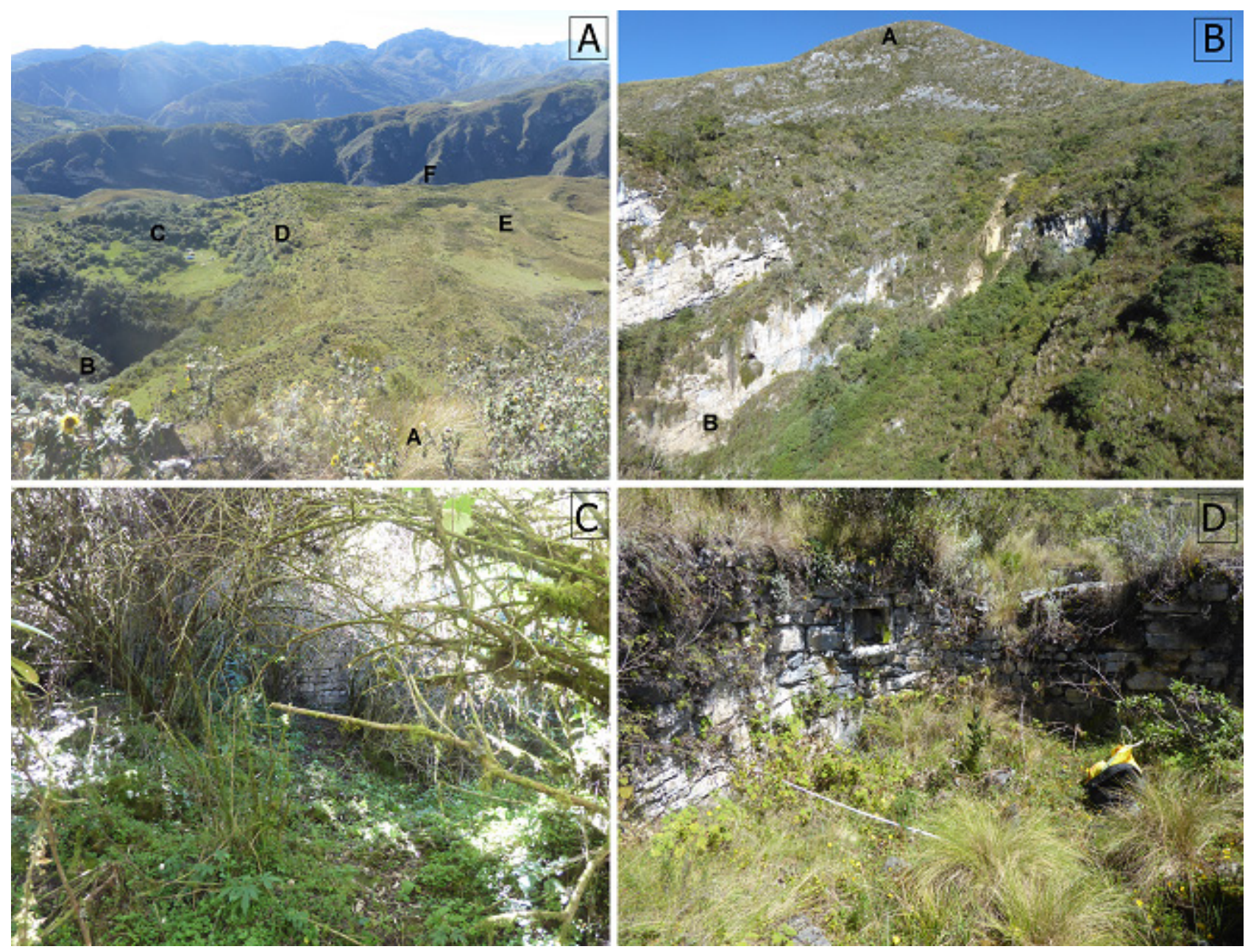

Figura 3. Representación fotográfica del complejo arqueológico de La Boveda (Distrito de Leymebamba, Provincia de Chachapoyas, Departamento de Amazonas, Norte de Perú). A. A. Cima de montaña, B. Vegetación en hundimiento kárstico profundo, C. Vegetación en ruinas arqueológicas con densa cobertura arbórea, D. Vegetacion en ruinas arqueológicas expuestas, E. Pajonales con influencia de fuego y vegetación en hundimientos kársticos, F. Vegetación de quebradas ocasionalmente secas y vegetación arbustiva en zona de riscos. B. Fotografía tomada de la parte central de La Boveda mirando hacía la montaña sur donde A. Cima de montaña y B. Vegetación en hundimiento kárstico profundo. C. Edificaciones en La Boveda, especificamente en zonas con alta cobertura arbórea. D. Edificación circular ubicada en el centro del complejo arqueológico de La Bóveda, esta zona ha sido considerada como vegetación en ruinas arqueológicas expuestas. Fotografías por DBMT, tomadas entre el 18 y 23 Junio 2018. 

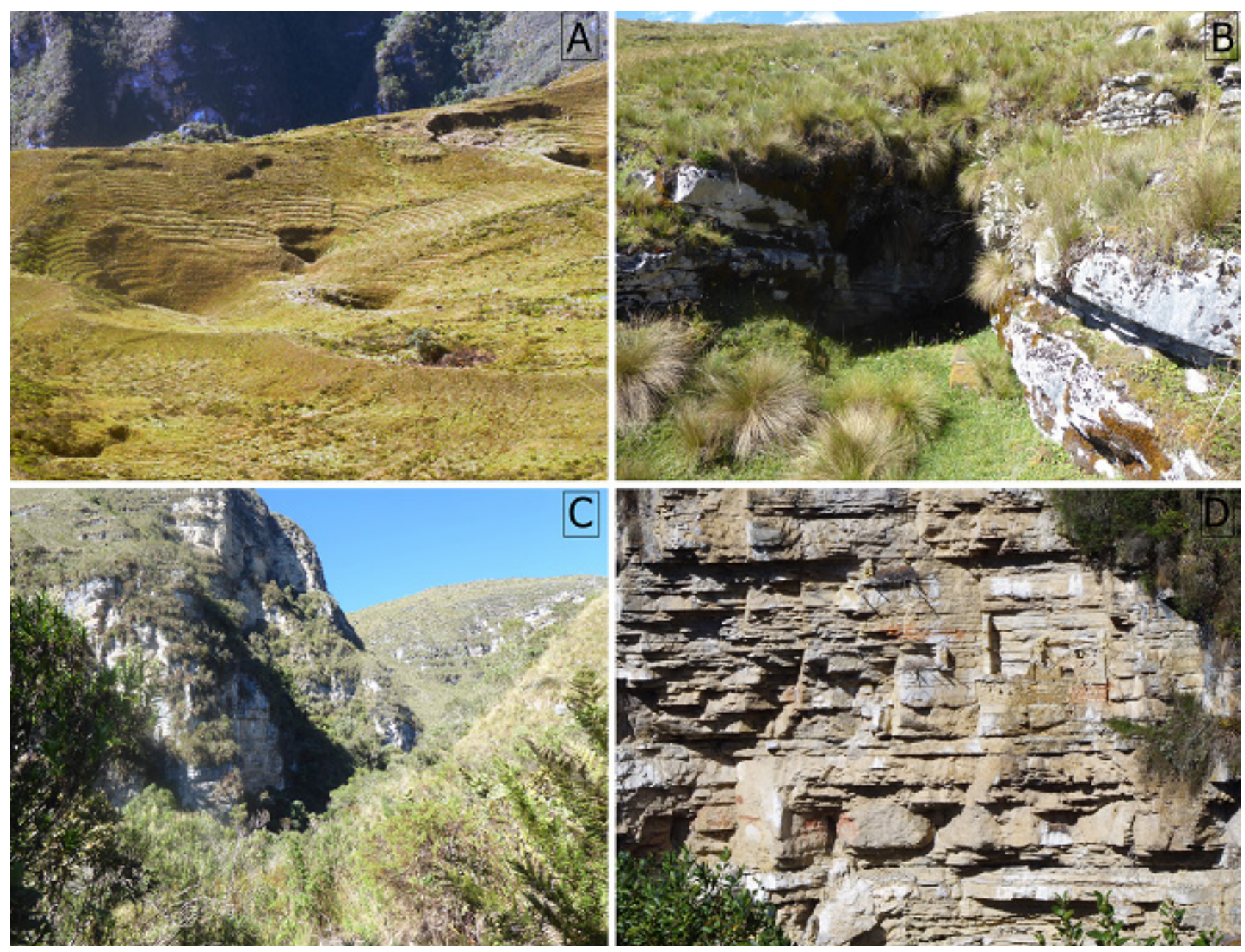

Figura 4. Representación fotográfica del complejo arqueológico de La Boveda (Distrito de Leymebamba, Provincia de Chachapoyas, Departamento de Amazonas, Norte de Perú). A. Andenería circular en los alrededores de diversos hundimientos kársticos donde se denotó evidencia de carbón por recientes incendios en la zona. B. Hundimiento kárstico tipo caverna rodeado de pajonales. C. Vegetación arbustiva y arbórea en quebradas ocasionalmente secas, el sector se denomina Diablo Wasi. D. Evidencia de construcciones Chachapoya en riscos en el sector de Diablo Wasi. Fotografías por DBMT, tomadas entre el 18 y 23 Junio 2018. 

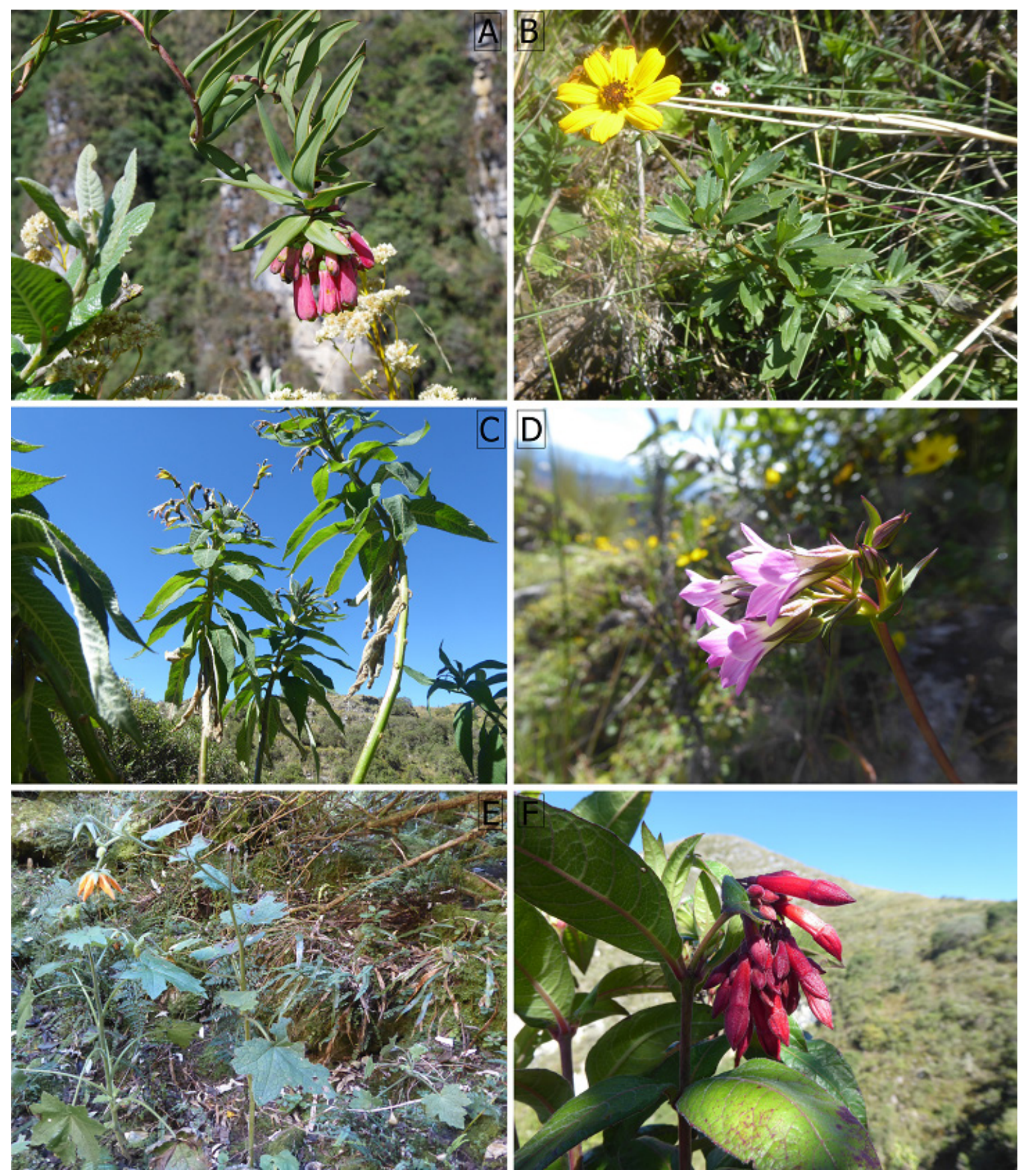

Figura 5. Muestra fotográfica de algunas especies endémicas halladas en el complejo arqueológico de La Boveda (Distrito de Leymebamba, Provincia de Chachapoyas, Departamento de Amazonas, Norte de Perú). A. Bomarea goniocaulon Baker (Alsmtroemeriaceae). B. Coreopsis lopez-mirandae Sagást. (Asteraceae). C. Siphocampylus matthiaei A. DC. (Campanulaceae). D. Gentianella decemnectaria J.S. Pringle (Gentianaceae). E. Nasa basilica T. Henning \& Weigend (Loasaceae). F. Fuchsia mathewsii J.F. Macbr. (Onagraceae). Fotografías por DBMT, tomadas entre el 18 y 23 Junio del 2018. 
yor riqueza de especies endémicas son: Asteraceae (23 especies, 41,1\%), Calceolariaceae (5 especies, 8,9\%), Caryophyllaceae, Lamiaceae (3 especies cada una, 5,4\%), Bromeliaceae, Orobanchaceae, Piperaceae (2 especies cada una, 3,6\%), Alstroemeriaceae, Campanulaceae, Crassulaceae, Fabaceae, Gentianaceae, Geraniaceae, Loasaceae, Melastomataceae, Onagraceae, Orchidaceae, Poaceae, Pteridaceae, Rosaceae, Scrophulariaceae, Urticaceae y Verbenaceae (una especie cada una, 1,8\%). Los géneros con mayor número de endemismos son: Calceolaria (Calceolariaceae) con 5 especies, Senecio (Asteraceae) con 4 especies, Baccharis y Gynoxys (Asteraceae) con 3 especies cada una, Coreopsis, Dendrophorbium (Asteraceae), Drymaria (Caryophyllaceae), Salvia (Lamiaceae), Castilleja (Orobanchaceae) y Peperomia (Piperaceae) con dos especies cada una.

\section{DISCUSIÓN}

En el complejo arqueológico de La Boveda (Distrito de Leymebamba, Provincia de Chachapoyas, Departamento de Amazonas, Norte de Perú), la vegetación está compuesta por 294 especies que representa aproximadamente el $4,2 \%$ de las especies de plantas registradas en el Departamento de Amazonas y el $14,7 \%$ de para la Provincia de Chachapoyas (Tropicos 2020).

En comparación con la vegetación del complejo arqueológico de Kuélap (Leiva et al., 2019), la similitud florística es muy baja, con menos del $10 \%$ de similitud de especies compartidas con respecto a la flora hallada en el complejo arqueológico de La Boveda, esto se debe a que aparentemente, la Jalca presenta mayor diversidad en zonas que no han sido intervenidas por el hombre.

Las familias mejor representadas en el complejo arqueológico de La Boveda por su alto número de especies son Asteraceae que representa el 25,2\%, seguido de Poaceae con 6,5\%, Caryophyllaceae con $4,4 \%$ y Polypodiaceae con $4,1 \%$.
Los pajonales por presentar espacios abiertos dominados por gramíneas, la diversidad vegetal está representada por 185 especies equivalente al $63 \%$ del total hallado para el complejo arqueológico. Por otro lado, las zonas boscosas presentaron la mayor diversidad vegetal compuesta por 264 especies equivalente al $90 \%$ del total de flora documentada.

La diversidad vegetal puede actuar como indicador de calidad de suelo y agua y por consiguiente ser utilizados en los procedimientos de restauración ecológica de corto, mediano y largo plazo. En este sentido, es importante destacar la riqueza florística que posee el complejo arqueológico de La Boveda, ya que las plantas forman un componente biológico propio del ecosistema de Jalca que se encuentra seriamente amenazado por la acción del hombre. En tal sentido, se confirma lo dicho por Tovar et al. (2012) en cuanto a que la fragmentación y pérdida del hábitat son consideradas como grandes amenazas sobre la biodiversidad, especialmente en ecosistemas tropicales montañosos. De igual forma, se contrastan los resultados con lo propuesto por Rodríguez (1996) y Marcelo-Peña et al. (2006) donde la desaparición de los ecosistemas del norte de Perú estan seriamente afectados por la deforestación y la quema de montes para conversión a agricultura lo cual genera la erosión de suelos y pérdida de habitat.

La invasión de "Chilca" (Baccharis latifolia) en la Jalca del departamento de Amazonas representa una seria amenaza a la vegetación nativa debido a su rápida adaptación al medio, especialmente en áreas que están siendo seriamente afectadas por incendios y conversión de campos a agricultura. Algunas especies del género Baccharis son conocidas por representar una sería amenaza en diversos ecosistemas de ambos hemisferios por su rápida adaptación a distintos medios (Caño et al., 2013). 


\section{CONCLUSIONES}

Como conclusión general, se da a conocer la composición de comunidades vegetales de la Jalca en el sur del Departamento de Amazonas (Perú) en ecosistemas dominados por gramíneas, hierbas, arbustos y árboles y cuya diversidad florística está conformada por 294 especies vasculares y helechos, que corresponden a 190 géneros y 65 familias.

Como parte de este estudio, se han seleccionado algunas especies arbóreas que podrían haber formado parte de los extensos bosques que una vez habitaron en la los alrededores del complejo arqueológico de la Boveda, siendo estos Escallonia sp. (Escalloniaceae), Buddleja incana Ruiz \& Pav. (Loganiaceae), Hesperomeles cuneata Lindl, Hesperomeles ferruginea (Pers.) Benth. y Polylepis multijuga Pilg. (Rosaceae). Dichas especies también pudieron ser de uso habitual entre los Chachapoya para edificación u otros usos pero no se puede confirmar a la fecha esta propocisión.

Es urgente invocar a la comunidad en general que se detengan los incendios en la zona ya que afectan gravemente los ecosistemas andinos (tanto a flora como a fauna) e incrementan las posibles incidencias que ocasionan el cambio climático por la emisión de gases nocivos a la atmósfera.

La mayoría de zonas evaluadas comprenden endemismos, en algunos casos con valores elevados, vinculado a este resultado se recomienda establecer planes de conservación en aquellas áreas que presentan altos índices de diversidad biológica.

\section{AGRADECIMIENTO}

El trabajo de campo fue realizado gracias a Anna Guengerich y fue auspiciado por la National Geographic (Grant Number HJ-090R-17). A John S. Ingham, quien dio la oportunidad de visitar la región estudiada por primera vez. A la población y autoridades de las localidades de Leymebamba y Tajopampa (Provincia de Cha- chapoyas) en el departamento de Amazonas. Las expediciones en Amazonas fueron apoyadas por S.P. Aguilar, E.A. Burgos, E.A. Chávez, R. Dover, H. Núñez del Prado, A. Peerson, L. Pratt, V. Slotten, E.M. Valle, E. Vega, G. Vega. Gracias a R. Bussmann, A. Damian, M.O. Dillon, W. Galiano, H. Gutierréz, G. Heiden, T. Henning, S. Knapp, E. Ortiz, G. Pino, J. Pringle, I. ReviIla, H. Trinidad, M. Weigend por el apoyo con la identificación de especies. Al Servicio Nacional Forestal y de Fauna Silvestre (SERFOR, Ministerio de Agricultura y Riego) por los permisos de colecta.

\section{REFERENCIAS BIBLIOGRÁFICAS}

Arfè, G., Mondillo, N., Boni, M., Balassone, G., Joachimski, M., Mormone, A., y Di Palma, T. (2017) The karst-Hosted Mina grande nonsulfide zinc deposit, Bongará district (Amazonas region, Peru). Economic Geology, 112 (5), 1089-1110.

Auler, A.S. (2017) Hypogene caves and karst of South America. En: Klimchouk, A., Palmer, A., De Waele, J., Auler, A., Audra, P. (Eds.), Hypogene Karst Regions and Caves of the World, Cave and Karst Systems of the World, vol. 2017 Springer International Publishing, Cham.

Beresford-Jones, D., Alarcón, C., Arce, S., Chepstow-Lusty, A., Whaley, O., Sturt, F., Gorriti, M., Portocarrero, O., y Cadwallader, L. (2009) Ocupación y subsistencia del Horizonte Temprano en el contexto de cambios ecológicos de largo plazo en las cuencas de Samaca y Ullujaya, valle bajo de Ica. Boletín de Arqueología PUCP, 13, 237-257.

Brako, L., y Zarucchi, J. (1993) Catalogue of the Flowering Plants and Gymnosperms of Peru. Monographs in Systematic Botany from the Missouri Botanical Garden, 45: 1-1286.

Caño, L., Campos, J., García-Magro, D., y Herrera, M. (2013) Replacement of estuarine communities by an exotic shrub: distribution and invasion history of Baccharis halimifolia in Europe. Biological invasions, 15 (6), 1183-1188. 
Cárdenas-Martin, M., y Milla-Villena, C. (1980) El desierto de Sechura en la arqueología peruana. Revista de la Universidad Católica, 8, 107-117.

Church, W., y Guengerich, A. (2017) La (re) construcción de Chachapoyas a través de la historia e histografía. Boletín de Arqueología PUCP, 23, 5-38.

Damián, A. (2019) Crocodeilanthe chachapoyensis (Orchidaceae: Pleurothallidinae), a new species from Amazonas, Peru. Finnish Zoological and Botanical Publishing Board. Annales Botanici Fennici, 56 (4-6), 301-304.

Escobedo, R. (2010) Fisiografía, informe temático. Proyecto Mesozonificación Ecológica y Económica para el Desarrollo Sostenible de la selva de Huánuco. Convenio entre el IIAP, DEVIDA. Iquitos, Perú.

Fernández-Hilario, R., Villanueva-Espinoza, R., Fack, V., Shanee, S., y Marcelo-Pena, J. (2020) Dos nuevas especies de Magnolia de los extremadamente amenazados bosques montanos del norte de Perú. Brittonia. [en línea], Disponible en <https://doi. org/10.1007/s12228-020-09607-y>

Galán de Mera, A., Linares-Perea, E., Martos-Rodríguez, F., Montoya-Quino, J., Rodríguez-Zegarra, C., Torres-Marquina, I., Trujillo-Vera, C., Villasante-Benavides, F., Árias-Gámez, J., y Vicente-Orellana, J. (2020) Distribution of archaeological sites of Ancient Peru is linked to climatology and natural vegetation. Plant Biosystems-An International Journal Dealing with all Aspects of Plant Biology, 1-28.

Guengerich, A. (2014) The architect's signature: The social production of a residential landscape at Monte Viudo, Chachapoyas, Peru. Journal of Anthropological Archaeology, 34, 1-16.

Hill, M. (1979) Twinspan, a Fortran program for arranging multivariate data in an ordered two way table by classification of the individuals and the attributes. Cornell University, Department of Ecology and Systematics, Ithaca, New York, US.

IPNI. (2014) The International Plant Names Index. [en línea], Disponible en <http://www. ipni.org> [Consulta: 2018 y 2020].
JSTOR. (2013) JSTOR Plant Science. [en línea], Disponible en <http://plants.jstor.org/> [Consulta: 2018 y 2020].

Kauffmann Doig, F. (1980) Los Pinchudos: exploración de ruinas intactas en la selva. Boletín de Lima, 7, 26-31.

Kauffmann Doig, F. (2009) Los Chachapoyas. Constructores de Kuélap y Pajatén. Centro de Investigaciones Turismo Kuelap S.A. (Lima, Perú).

Kauffmann Doig, F. (2017) Chachapoyas Culture. Roberto Gheller D. Editores (Lima, Perú).

Knapp, R. (1984) Considerations on quantitative parameters and qualitative attributes in vegetation analysis and in phytosociological relevés. In: Knapp, R. (ed.) Sampling methods and taxon analysis in vegetation science, 77-100.

Leiva, S., Rodríguez, L., Pollack, J., Briceño, J., Jiménez, G., Gayoso, I., Saldaña, M., Barrena, E., Pariente, W., Gosgót, O., Gamarra y Rascón. (2019) Diversidad natural y cultural del Complejo Arqueológico Kuélap (provincia Luya, región Amazonas): la fortaleza de los hombres de las nubes. Arnaldoa, 26 (3), 883-930.

León, B., Roque, J., Ulloa, C., Jorgensen, P., Pitman, N. y Cano, A. (2006) Libro Rojo de las plantas endémicas del Perú. Revista Peruana de Biología 13, 946-965.

Marcelo-Peña, J., Sánchez-Vega, I., y Millán-Tapia, J. (2006) Estado actual de la diversidad floristica del paramo sectores: el espino y palambe, sallique, jaen. Cajamarca. Perú. Ecología Aplicada, 5 (1-2), 1-8.

Martorell, J. (1975) Las regiones kársticas del Perú. Datos para una exploración al Karst de Cutervo. [en línea], Disponible en <cuevasdelperu.org>

MINAM (2009) Indicadores Ambientales Amazonas. Serie - Indicadores Ambientales. Ministerio del Ambiente.

Montesinos-Tubée, D. (2016) Diversidad florística asociada a los restos arqueológicos de la cultura Yarowilca en los departamentos de Huánuco y Ancash, Perú. Arnaldoa 23 (2), 475-516.

Montesinos-Tubee, D. (2017a) Dendrophorbium chopinii (Compositae: Senecioneae), a 
new species from Amazonas Region, Peru. Phytotaxa, 313 (2), 210-216.

Montesinos-Tubée, D. (2017b) La Influencia Del Fuego Y Del Pastoreo En La Diversidad De La Flora Vascular Del Complejo Arqueológico De Chiquia, Distrito De Jesús, Provincia De Lauricocha, Departamento De Huánuco, Perú. Chloris Chilensis, 20, 2.

Montesinos-Tubée, D., Cano, A., García-Llatas, L., Ju, Y., y Kool, A. (2018) Paronychia sanchez-vegae (Caryophyllaceae), a new woody species of Paronychia from North Peru. Phytotaxa, 334 (1), 41-48.

Moonlight, P., Hollands, R., Cano, A. y Purvis, D. (2020) A new species of tuberous Begonia (Begoniaceae) from Andean Peru. Edinburgh Journal of Botany, 77 (1), 145-159. [en línea], Disponible en <doi:10.1017/ S0960428619000301>

Mueller-Dombois, D., y Ellenberg, H. (1974) Aims and methods of vegetation ecology. John Wiley y Sons. 45-66.

Rodríguez, L. (1996) Diversidad Biológica del Perú. Zonas Prioritarias para la Conservación. PROYECTO FANPE GTZ-INRENA. Lima - Perú.

Rodríguez, E., Monzón, K., Martínez, B., Liza, V., Morillo, M., Bernabé, L., y Mora, M. (2015) Comunidades vegetales del Complejo Arqueológico Chan Chan, provincia Trujillo, región La Libertad, Perú. Arnaldoa, 22 (1), 119-138.

Romero-Hernández, C., Téllez-Valdés, O., y Bussmann, R. (2019) Dioscorea chusqueifolia (Dioscoreaceae), a new species from northern Peru. Brittonia, 71 (4), 353-358.

Rostworowski de Diez Canseco, M. (1981) Recursos naturales renovables y pesca, siglos XVI y XVII. Instituto de Estudios Peruanos, Lima.

Schjellerup, I., Sorenson, M., Espinoza, C., Quipuscoa, V., y Peña, V. (2003) Los Valles Olvidados: Pasado y Presente en la Utilizatión de Recursos en la Ceja de Selva, Perú. Ethnographic Monographs, № 1.

Schjellerup, I. (2017) La provincia inka de Chachapoyas. Boletín de Arqueología PUCP, 23, 259-282.
SENAMHI (2018) Servicio Nacional de Meteorología e Hidrología del Perú. Información meteorológica de las estaciones de Huacaybamba y Leymebamba-Chachapoyas para los periodos 2017-2018. Lima, Perú.

Thiers, B. (2016) Index herbariorum. A global directory of public herbaria and associated staff. New York Botanical Garden. [en línea], Disponible en <http://sweetgum.nybg.org/ ih/> (Accedido en Abril 2020).

Torres, E., Albán, J., y Muñoz, A. (2018) Estudio etnobotánico de plantas medicinales utilizadas en comunidades adyacentes al Área de Conservación Privada San Antonio, Chachapoyas, Amazonas, Perú. Revista Científica UNTRM: Ciencias Naturales e Ingeniería, 1 (1), 65-73.

Tovar, C., Duivenvoorden, J., Sánchez-Vega, I., y Seijmonsbergen, A. (2012) Recent Changes in Patch Characteristics and Plant Communities in the Jalca Grasslands of the Peruvian Andes. Biotropica, 44 (3), 321-330.

Toyne, J., y Anzellini, A. (2017) Sociedad, identidad y variedad de los mausoleos de La Petaca, Chachapoyas. Boletín de Arqueología PUCP, 23, 231-257.

Tropicos. (2020) Missouri Botanical Garden. [en línea], Disponible en <http://tropicos.org.> [Accedido entre 2018 y 2020].

Von Hagen, A. (2016) Los Chachapoya y la Laguna de los Cóndores. Museo Leymebamba, Amazonas, Perú.

Weberbauer, A. (1945) El mundo vegetal de los Andes Peruanos. Estación Experimental Agrícola de la Molina. Dirección de Agricultura, Lima.

Wernke, S. (2003) An Archaeo-history of Andean Community and Landcape: The Late Prehispanic and Early Colonial Colca Valley, Peru. University of Wisconsin-Madison.

\section{Conflicto de interés}

Declaro que esta investigación y publicación no tiene conflictos de interés. 
